\title{
The Influences of Soft and Stiff Inclusions on the Mechanical Properties of Cementitious Composites
}

\author{
Gabriel Falzone $\left({ }^{*},{ }^{\dagger}\right)$, Guillermo Puerta Falla $\left({ }^{\ddagger}\right)$, Zhenhua Wei $\left({ }^{\S}\right)$, Mingjie Zhao $\left(^{* *}\right)$, Aditya Kumar \\ $\left({ }^{\dagger+}\right)$, Mathieu Bauchy $\left({ }^{\ddagger \ddagger}\right)$, Narayanan Neithalath $\left({ }^{\S \S}\right)$, Laurent Pilon $\left({ }^{* * *}\right)$ and Gaurav Sant $\left({ }^{\dagger+\dagger},{ }^{\ddagger \ddagger}\right)$
}

\section{Abstract}

The embedment of microencapsulated phase change materials (PCM) is a promising means for improving the thermal inertia of concrete. However the addition of such soft microcapsules degrades the mechanical properties, i.e., the elastic moduli and compressive strength, of cement-based composites. This study experimentally quantifies the effects of stiff quartz inclusions and soft PCM microcapsules, individually, and when added together, on the mechanical properties of cementitious composites. In addition, a variety of effective medium approximations (EMAs) were evaluated for their ability to predict the experimentally measured composite effective moduli. The EMAs proposed by Hobbs and Garboczi and Berryman (G-B) reliably estimate experimental data. The experimental data and the EMAs were applied to develop a design rule for performance equivalence, such that the composite modulus of elasticity can be maintained equivalent to that of the cementitious paste matrix, in spite of the addition of soft PCM microcapsules.

Keywords: Elasticity; Mechanical properties; Micromechanics; Mechanical testing; Modeling

\footnotetext{
${ }^{*}$ Research Assistant (Corresponding Author), Laboratory for the Chemistry of Construction Materials(LC $\left.{ }^{2}\right)$, Department of Civil and Environmental Engineering, University of California, Los Angeles, CA, USA

${ }^{\dagger}$ Research Assistant, Department of Materials Science and Engineering, University of California, Los Angeles, CA, USA, Email: gabefalzone@gmail.com

${ }^{\ddagger}$ Research Assistant, Laboratory for the Chemistry of Construction Materials $\left(\mathrm{LC}^{2}\right)$, Department of Civil and Environmental Engineering, University of California, Los Angeles, CA, USA, Email: gpuertafalla@ucla.edu

${ }^{\S}$ Research Assistant, Laboratory for the Chemistry of Construction Materials $\left(\mathrm{LC}^{2}\right)$, Department of Civil and Environmental Engineering, University of California, Los Angeles, CA, USA, Email: zhenhuawei@ucla.edu

${ }^{* *}$ Research Assistant, Laboratory for the Chemistry of Construction Materials $\left(\mathrm{LC}^{2}\right)$, Department of Civil and Environmental Engineering, University of California, Los Angeles, CA, USA, Email: jameszhao217@gmail.com

${ }^{++}$Scientist, Laboratory for the Chemistry of Construction Materials $\left(L C^{2}\right)$, Department of Civil and Environmental Engineering, University of California, Los Angeles, CA, USA, Email: adityaku@ucla.edu

${ }^{\ddagger \ddagger}$ Assistant Professor, Laboratory for Physics of Amorphous and Inorganic Solids (PARISlab), Department of Civil and Environmental Engineering, University of California, Los Angeles, CA, USA, Email: bauchy@gmail.com

${ }^{\S \S}$ Associate Professor, School of Sustainable Engineering and the Built Environment, Arizona State University, Tempe, AZ, USA, Email: narayanan.neithalath@asu.edu

${ }^{* * *}$ Professor, Department of Mechanical and Aerospace Engineering, University of California, Los Angeles, CA, USA, Email: pilon@seas.ucla.edu

${ }^{+++}$Associate Professor and Henry Samueli Fellow (Corresponding Author), Laboratory for the Chemistry of Construction Materials(LC ${ }^{2}$ ), Department of Civil and Environmental Engineering, University of California, Los Angeles, CA, USA, Email: gsant@ucla.edu

${ }^{\ddagger \ddagger \ddagger}$ Member, California Nanosystems Institute (CNSI), University of California, Los Angeles, CA, USA
} 


\section{Introduction}

Cementitious composites consist of hard mineral inclusions embedded within a matrix that is dominantly composed of calcium silicate hydrates [1]. Inclusions are embedded in cementing composites to reduce cost, since mineral aggregate inclusions (i.e., stone and sand) are far less expensive than cement, or to alter performance, such as to improve their stiffness [2]. There is interest in embedding microencapsulated phase change materials (PCM) to concrete to improve its thermal inertia, e.g., for building envelope applications. Indeed, based on their ability to store and release heat, PCMs increase the thermal inertia of building materials, and can thus improve the energy efficiency of buildings [3-8]. More recently, Fernandes et al. [9] also demonstrated the ability of PCM inclusions to reduce thermal cracking in cementitious systems. In such cases, it has been noted that while the addition of PCM improves thermal performance, it degrades the composite's mechanical properties (i.e., both elastic modulus and compressive strength) [9].

Several studies have noted that the compressive strength of cementitious materials decreases with increasing PCM content [9-11]. However, data on the influence of PCMs on the stiffness of cementitious composites are far less available. Reductions in the mechanical properties are problematic, as for reasons of structural capacity, even in building envelope applications, it is necessary that cement-based composites demonstrate sufficient strength and stiffness. Since PCMs are soft inclusions, i.e., substantially weaker than the inorganic cementing matrix, parallels can be drawn to the additions of other soft inclusions to cementitious systems, e.g., of rubber inclusions, EPS (expanded polystyrene) inclusions etc., which may be added to reduce the density of concrete [12-20]. While these studies indicate similar trends as in the case of PCM additions, i.e., the mechanical properties degrade with increasing inclusion content, they seldom present complete experimental data of elastic moduli $(E, K$, or $G$ ) and less often utilize EMAs to describe composite properties in relation to inclusion characteristics. Thus, this study has three main aims:

- To experimentally quantify the reduction in stiffness (modulus of elasticity, $E$ ) that is caused due to the addition of microencapsulated PCMs to cementitious mortars and to assess the success of the countermeasure of adding stiff (i.e., quartz) inclusions,

- To critically assess the ability of EMAs to predict the modulus of elasticity of cementitious materials containing both soft and stiff inclusions. While such studies have often been carried out for the case of inclusions stiffer than the matrix, far fewer studies have considered the case of both stiff and soft inclusions embedded simultaneously, and,

- To develop design rules which permit the formulation of composites with a modulus of elasticity that is equivalent to that of the paste matrix, even when soft inclusions are added, with due consideration of the properties/dosages of matrix and inclusion components. 


\section{Background}

\subsection{Experimental studies of the mechanical properties of cementitious composites} Experimental studies of cementitious composites containing soft inclusions have frequently relied on measurements of compressive strength as an indication of mechanical properties. Eldin and Senouci [13] measured the compressive strength of concretes containing tire rubber added by replacement of mineral aggregate. Their experimental data fit the empirical model of Popovics [21], which predicts an exponential decay in the compressive strength with increasing inclusion volume fraction. Le Roy et al. [22] presented compressive strength data of concrete containing EPS beads. The data were fit to a hyperbolic function that accounted for the maximum packing fraction of EPS, which was modified by an empirical "fitting factor". Hunger et al. [10] measured the compressive strength of concretes containing PCMs and observed a $13 \%$ decrease in strength for each $1 \%$ increase in PCM dosage (by mass of concrete). Cui et al. [23] noted a $1 \%$ decrease in compressive strength for each $1 \%$ increase in PCM mass percentage in a cement paste matrix.

The influence of soft inclusions on the effective modulus of elasticity of cementitious composites has been far less frequently evaluated. Ghaly and Gill [24] measured the modulus of elasticity of concrete containing plastic chips at various water-to-cement ratios (w/c, by mass). The reduction in the modulus of elasticity due to plastic inclusions was presented as a function of the percent area of plastic inclusions measured in 2D slices. Their data was fit to an exponential relation, but the scatter in experimental data was large. Ganjian et al. [25] measured the modulus of elasticity of concretes containing scrap tire rubber. They observed a decrease in the modulus as a function of rubber volume fraction, but no attempt was made to describe these trends by fitting models or by the use of effective medium approximations. Shin et al. [20] presented modulus of elasticity data of cement mortars containing EPS inclusions dosed in partial replacement of normal weight sand inclusions. Xu et al. [26] noted an approximately linear decrease in the measured modulus of elasticity of EPS-containing concretes, and fit the data to an empirical polynomial relation. While these studies present valuable experimental data of the influence of soft inclusions on effective moduli, the mixture designs considered are not conducive to assessing the simultaneous effect of the addition of stiff inclusions. This is because they generally utilized the mortar (cement paste + stiff inclusions) as a homogeneous matrix component. In summary, while these studies clearly highlight the negative effects of soft inclusions on the mechanical response of concretes, the relations developed do not offer quantitative guidelines for predicting composite properties based on the mixture proportions and component properties, as they require fitting to empirical data, and are often mixture specific. 


\subsection{Effective medium approximations and their application to cementitious composites}

Concrete comprises inclusions embedded in a cement paste matrix. Due to the formation of a water-rich zone around non-reactive inclusions, and wall-effects which hamper particle packing near flat surfaces, a surrounding layer of porous, and therefore weaker hydration products known as the interfacial transition zone (ITZ), develops around the aggregates [27]. This effect is independent of inclusion stiffness, but depends on particle size, i.e., a thicker ITZ forms around larger aggregates $[28,29]$. This results in cementitious microstructures consisting at a minimum of three material components, i.e., (i) the cement paste matrix, (ii) the aggregate inclusions, and (iii) the ITZ. While pores are present in the matrix (including in the ITZ) and aggregates, they are not considered as a discrete component, but rather their influence is lumped into the elastic response of the paste matrix and aggregates, respectively [30]. The addition of PCMs further complicates the organization of cementitious microstructures due to another component being added. To better evaluate these aspects, this section provides an overview of effective medium approximations (EMAs) for the elastic moduli of composite media and highlights how formulation details, e.g., the volume fractions, spatial distribution, and properties of inclusion, matrix and interfacial components may influence the property predictions [2]. In general, all EMAs discussed assume that the individual material components are: i) homogeneous, ii) isotropic, and iii) are (essentially) linear elastic.

The parallel (isostrain) and series (isostress) formulations developed by Voigt [31] and Reuss [32], respectively, can be applied to estimate the lower and upper bounds on the elastic moduli of two-component composites. Hansen [33] noted that the isostrain assumption is better when the stiffness of the matrix is greater than that of the inclusion, and the opposite for the isostress case. Hill [34] highlighted that the arithmetic mean of the Reuss and Voigt EMAs can be used as an approximation within these bounds, often referred to as the Reuss-Voigt-Hill (RVH) average.

Hashin and Shtrikman (H-S) [35] developed bounds for the elastic moduli of multi-component composites, which were proved to be the tightest bounds possible without any consideration of microstructural geometry [36]. Walpole's formulation of these bounds, which is readily extended to " $N$ " component composites, is expressed as [36]

$$
\begin{aligned}
& {\left[\sum_{r=1}^{N} \phi_{r}\left(K_{l}^{\prime}+K_{r}\right)^{-1}\right]^{-1}-K_{l}^{\prime} \leq K_{e f f} \leq\left[\sum_{r=1}^{N} \phi_{r}\left(K_{g}^{\prime}+K_{r}\right)^{-1}\right]^{-1}-K_{g}^{\prime}} \\
& {\left[\sum_{r=1}^{N} \phi_{r}\left(G_{l}^{\prime}+G_{r}\right)^{-1}\right]^{-1}-G_{l}^{\prime} \leq G_{e f f} \leq\left[\sum_{r=1}^{N} \phi_{r}\left(G_{g}^{\prime}+G_{r}\right)^{-1}\right]^{-1}-G_{g}^{\prime}}
\end{aligned}
$$


where: $K_{l}^{\prime}=\frac{4}{3} G_{l}, K_{g}^{\prime}=\frac{4}{3} G_{g}, G_{l}^{\prime}=\frac{3}{2}\left(\frac{1}{G_{l}}+\frac{10}{9 K_{l}+8 G_{l}}\right)^{-1}$, and $G_{g}^{\prime}=\frac{3}{2}\left(\frac{1}{G_{g}}+\frac{10}{9 K_{l}+8 G_{l}}\right)^{-1}$. In

136

137

138

139

140

141

142

143

144

145

146

147

148

149

150

151 and

this equation, $K_{/}$and $G_{l}$ are the least bulk and shear moduli of the components, while $K_{g}$ and $G_{g}$ are the greatest. The subscript " $r$ " refers to the $r^{\text {th }}$ component in the composite. Nilsen and Monteiro [37] observed that experimental elastic moduli of cementitious composites often lie below the H-S lower bound. They attributed this mismatch to the presence of the weaker ITZ and concluded that three-component EMAs must be used in such cases. While such aspects are relevant to the case of $\mathrm{PCM}$-containing concretes, the $\mathrm{H}-\mathrm{S}$ bounds are known to be very wide when the ratio of the stiffnesses of the individual components is greater than 10 [38]. Le Roy et al. [22] found that the Hashin-Shtrikman upper bound provided a reasonable fit to experimental modulus of elasticity data EPS-containing concrete.

Hobbs [39] derived EMAs for the Young's modulus for two cases: (a) when the Poisson's ratios of the components are identical, and (b) when there is considerable mismatch in Poisson's ratios and the inclusions are assumed to be voids (such that $E_{i} \approx 0$ ). The two resulting equations for the effective composite modulus of elasticity based on these assumptions are, respectively

$$
\begin{gathered}
E_{\text {eff }}=E_{m}\left[1+\frac{2 \phi_{i}\left(E_{i}-E_{m}\right)}{\left(E_{i}+E_{m}\right)-\phi_{i}\left(E_{i}-E_{m}\right)}\right] \\
E_{\text {eff }}=E_{m}\left[\frac{\left(1-2 v_{\text {eff }}\right)\left(1-\phi_{i}\right)}{\left(1-2 v_{m}\right)\left(1+\phi_{i}\right)}\right]
\end{gathered}
$$

152

153

154

155

156

157

158

159

160

161

162

163

164

165

166

where $E_{\text {eff }}$ is the effective composite modulus of elasticity, $E_{i}$ and $E_{m}$ are the moduli of elasticity of the inclusions and matrix. The volume fractions $\phi_{i}$ and $\phi_{m}$ of the inclusions and the matrix, respectively are such that $\phi_{i}+\phi_{m}=1$. The effective Poisson's ratio of the composite is $v_{\text {eff, }}$ while $v_{i}$ and $v_{m}$ are the Poisson's ratios of the inclusion and matrix. In Equation 2(b), it is unclear what value to assume for the effective Poisson's ratio of the composite. The Poisson's ratio is often calculated from the effective RVH-averaged bulk $(K)$ and shear $(G)$ moduli. However, the present study applies the RVH-averaged Poisson's ratio, given by

$$
v_{\text {eff }}=\left[\left(v_{i} \phi_{i}+v_{m} \phi_{m}\right)+\left(\frac{v_{i} v_{m}}{v_{i} \phi_{m}+v_{m} \phi_{i}}\right)\right] / 2
$$

This relation was selected as it yielded a more gradual transition in the Poisson's ratio with increasing PCM volume fraction, thereby offering better agreement with measured modulus of elasticity data (see also supplementary information, Section S1 for further discussion).

Rigorous EMAs for the composite properties require consideration of the geometrical features of inclusion dispersion in the composite to render accurate predictions. This is more so when the inclusions are softer than the matrix, and hence inclusion shape may be influential [40]. The 
167

168

169

170

171

172

173

174

175

176

177

178

179

180

181

182

183

generalized self-consistent method (GSCM) popularized by Christensen and Lo [41] is one such EMA for predicting elastic moduli. The effective shear modulus of the composite via the GSCM is obtained from the non-negative root of the equation [42]

$$
\alpha\left[\left(G_{\text {eff }} / G_{m}\right)-1\right]^{2}+\beta\left[\left(G_{e f f} / G_{m}\right)-1\right]+\gamma=0
$$

where the parameters $\alpha, \beta$, and $\gamma$ are dependent upon the Poisson's ratios of the inclusion and matrix, as well as the inclusion volume fraction [41]. Christensen [43] also detailed a formulation of the bulk modulus of the composite which is identical to the result of the composite sphere assemblage of Hashin [44]. Huang et al. [45] demonstrated a simple approximation for the GSCM for application to N-component composites.

Mori and Tanaka [46] developed a method for calculating the elastic moduli of a composite based on the concept of eigenstrains and 'average stress' in the matrix. For a composite of randomly distributed spherical inclusions at relatively large concentrations, the relevant MoriTanaka (M-T) equations for bulk and shear modulus are [46]

$$
\begin{gathered}
K_{e f f}=K_{m}+\frac{\phi_{i}\left(K_{i}-K_{m}\right)}{1+\left(1-\phi_{i}\right) \frac{K_{i}-K_{m}}{K_{m}+\frac{4}{3} G_{m}}} \\
G_{e f f}=G_{m}+\frac{\phi_{i}\left(G_{i}-G_{m}\right)}{1+\left(1-\phi_{i}\right) \frac{G_{i}-G_{m}}{G_{m}+F_{m}}}
\end{gathered}
$$

where, for spherical inclusions, $F_{m}=\left(\frac{G_{m}}{6}\right) \frac{9 K_{m}+8 G_{m}}{K_{m}+2 G_{m}}$. These equations can also be written for multi-component composites [47]. Weng [48] showed that the M-T formulation is equivalent to the $\mathrm{H}-\mathrm{S}$ upper or lower bounds of a composite containing spherical inclusions of either hard or soft homogeneous and isotropic particles, respectively. For other multicomponent composites, however, Norris [49] demonstrated that the bulk modulus predicted by the M-T scheme can exceed the H-S bounds for certain combinations of mechanical properties of the different constituents, e.g., in a three-component composite where the inclusion bulk moduli are two to three times greater than that of the matrix.

Kuster and Toksöz (K-T) [50] used a wave-scattering analogy to develop an EMA for the elastic moduli of composites composed of a continuous matrix containing randomly embedded, polydisperse, spherical homogeneous inclusions. Berryman and Berge [51] found that this EMA and that of Mori and Tanaka are accurate only when the inclusion volume fraction is less than 0.30. The multi-component K-T equations for bulk and shear modulus as written by Berryman and Berge [51] are given by

$$
\left(K_{\text {eff }}-K_{m}\right) \frac{K_{m}+\frac{4}{3} G_{m}}{K_{\text {eff }}+\frac{4}{3} G_{m}}=\sum_{i=1}^{N} \phi_{i}\left(K_{i}-K_{m}\right) P^{m, i}
$$




$$
\left(G_{e f f}-G_{m}\right) \frac{G_{m}+\frac{4}{3} F_{m}}{G_{e f f}+\frac{4}{3} F_{m}}=\sum_{i=1}^{N} \phi_{i}\left(G_{i}-G_{m}\right) Q^{m, i}
$$

200 where, for spherical inclusions, $P^{m, i}=\frac{K_{m}+\frac{4}{3} G_{m}}{K_{i}+\frac{4}{3} G_{m}}, Q^{m, i}=\frac{G_{m}+F_{m}}{G_{i}+F_{m}}$, and the subscript "i=1"

201

202

203

204

205

206

207

208

209

210

211

213

214

215

216

217

218

219

220

221

222

223

224

225

226

227

228

corresponds to the matrix. $F_{m}$ is the same as given in the M-T EMA (see Equation 4).

The differential effective medium theory (D-EMT) replaces an incremental volume fraction of the matrix with inclusion, such that the inclusion volume fraction can be approximated as dilute. The particle and matrix are homogenized, and the incremental replacement is repeated, such that large inclusion volume fractions can be incorporated into the effective matrix. This approach prevents phase inversion, which is necessary when the ratio of moduli between the components is large, e.g., in the case of air voids or soft inclusions. Scalar D-EMT equations for the bulk and shear moduli of a composite of isotropic spheres oriented in an isotropic matrix are given by [52]

$$
\begin{aligned}
& \frac{\mathrm{d} K_{e f f}}{\mathrm{~d} \phi_{i}}=\left[\frac{K_{i}-K_{e f f}}{1-\phi_{i}}\right]\left[\frac{K_{e f f}+K^{\prime}}{K_{i}+K^{\prime}}\right] \\
& \frac{\mathrm{d} G_{e f f}}{\mathrm{~d} \phi_{i}}=\left[\frac{G_{i}-G_{e f f}}{1-\phi_{i}}\right]\left[\frac{G_{e f f}+G^{\prime}}{G_{i}+G^{\prime}}\right]
\end{aligned}
$$

where, $K^{\prime}=\frac{4}{3} G_{e f f}, G^{\prime}=\left(\frac{G_{e f f}}{6}\right) \frac{9 K_{e f f}+8 G_{e f f}}{K_{e f f}+2 G_{e f f}}$; and $K_{e f f}=K_{m}$ and $G_{e f f}=G_{m}$ at $\phi_{i}=0$. These equations form a set of coupled non-linear ordinary differential equations, solutions to which render the elastic moduli of the two-component composite. These equations can be solved by a $4^{\text {th }}$ order Runge-Kutta scheme [53]. Miled et al. [54] found that the differential scheme best predicted the effective modulus of elasticity of EPS-mortar specimens over a large range of EPS volume fractions. However, their experiments were not designed to describe the combined influences of soft and stiff inclusions in cementitious composites, as the matrix constituted the combination of the stiff aggregates and cement paste.

Due to the existence of the ITZ in cementitious composites, it is necessary to consider a shell of ITZ around the inclusions as part of an "effective particle." Garboczi and Berryman (G-B) [55] utilized the GSCM to homogenize an effective particle of aggregate and its ITZ, which was then homogenized into the cement-paste matrix via D-EMT. The use of D-EMT is well-suited to the microstructure of cementitious composites, as their aggregates are always discrete particles in a continuous matrix. However, D-EMT exhibits a dependency on the order in which inclusions are embedded into the matrix when the inclusions may have differing properties, i.e., the 
results may change whether the stiff or soft inclusion is first homogenized into the matrix component, when multiple aggregate components are present [56]. Therefore, GSCM is utilized, considering 3D spherical particles (inclusions), to simplify the concrete microstructure into two components (i.e., effective particle including the aggregate + ITZ, and cement paste). GSCM considers concentric spheres (e.g., core + shell) and is therefore ideal for homogenization of effective inclusion-shell particles. This method has been shown to agree very well with both experimental data and results obtained via the finite element method [55] for cementitious composites.

\subsection{Comments on the elastic properties of the interfacial transition zone (ITZ)}

Consideration of the ITZ component in EMAs requires knowledge of its elastic properties and volume fraction in concrete. Accounts from the literature typically assign the ITZ a modulus of elasticity ranging between $50 \%$ and $80 \%$ that of the bulk cement paste matrix [57]. Here, the ITZ was assigned a modulus of elasticity $50 \%$ that of the matrix, i.e., $E_{I T Z}=0.5 \cdot E_{m}$, for all inclusion-containing composites [58]. The Poisson's ratio of the ITZ was assumed equivalent to that of the paste matrix $[57,59,60]$. Although the ITZ "in reality" demonstrates a gradient of properties as a function of distance from the surface of an encompassed aggregate particle, it is often modeled as a homogeneous section with a fixed thickness $[55,61,62]$. Therefore, the ITZ thickness around quartz particles was assumed independent of the particle diameter and taken as $t_{I T Z, q}=10 \mu \mathrm{m}$, which represents the lower bound of experimental observations of ITZ thickness (around 10-50 $\mu \mathrm{m}$ ) [61,63-65]. The quartz particles were assumed to be spherical with a size equal to their median diameter $\left(d_{50, q}\right.$, Figure 1$)$. This assumption is justified due to the small elastic mismatch between the elastic moduli of the quartz inclusion and the cement paste matrix $\left(0.13 \leq E_{m} / E_{q} \leq 0.23\right)$, as a function of matrix age. Indeed, this tends to minimize the influence of inclusion shape [66-68]. These properties produce a relation for the ITZ volume fraction, $\phi_{I T Z, q}=\left(\left(t_{I T Z, q}+d_{50, q} / 2\right)^{3} /\left(d_{50, q} / 2\right)^{3}-1\right) \cdot \phi_{q}$ for a given quartz volume fraction $\phi_{q}$. For example, this equation yields an ITZ volume fraction $\phi_{I T Z, q}=0.025$ for a quartz inclusion volume fraction $\phi_{q}=0.30$ and a median diameter $d_{50, q}=365 \mu \mathrm{m}$.

Few, if any studies have discussed the existence and properties of ITZs around particles as small as the PCM microcapsules used in the present study $[69,70]$. As the PCM microcapsules were only slightly larger than the cement grains $\left(d_{50, p} \approx 20 \mu \mathrm{m}\right)$, they were expected to exhibit a reduced wall effect in comparison to quartz inclusions $[71,72]$. A simple assumption to account for this is that the ITZ thickness scales linearly with microcapsule size, i.e., $t_{I T Z, p}=C \cdot r_{p}$, where $C$ $=0.25$ and $r_{p}$ is the PCM microcapsule radius $(\mu \mathrm{m})$. The value of $C$ was selected such that the ITZ thickness at the maximum PCM microcapsule size matched that of the quartz particles. As a result of this assumption of the ITZ thickness, the volume fraction of the PCM-related ITZ in the composite followed a radius-independent relation that is given by $\phi_{I T Z, p}=\left(C^{3}+3 C^{2}+3 C\right) \cdot \phi_{p}$. As 
such, for a PCM volume fraction $\phi_{p}=0.30$, the associated ITZ volume fraction within the composite was predicted as $\phi_{I T Z, p}=0.29$ when $C=0.25$. This implies that the cement paste matrix which accounted for 0.70 of the composite volume, included 0.29 ITZ, as a volume fraction, with the residual 0.41 volume fraction being a bulk matrix phase, free of ITZ induced inhomogeneities. These assumptions were used throughout the study.

\section{Materials and Experimental Methods}

An ASTM C150 [73] compliant Type I/II ordinary portland cement (OPC) was used. The OPC had a nominal mineralogical composition of: $56.5 \% \mathrm{Ca}_{3} \mathrm{SiO}_{5}, 18.0 \% \mathrm{Ca}_{2} \mathrm{SiO}_{4}, 11.4 \% \mathrm{Ca}_{4} \mathrm{Al}_{2} \mathrm{Fe}_{2} \mathrm{O}_{10}$, 6.3\% $\mathrm{Ca}_{3} \mathrm{Al}_{2} \mathrm{O}_{6}, 4.6 \% \mathrm{CaCO}_{3}$, and $1.1 \% \mathrm{CaSO}_{4} \cdot 2 \mathrm{H}_{2} \mathrm{O}$. A microencapsulated PCM, MPCM-24D produced by Microtek Laboratories Inc. was used as a soft inclusion. The PCM consisted of a paraffin core encapsulated within a melamine-formaldehyde (MF) shell. Graded quartz sand, compliant with ASTM C778 [74] was used as a stiff inclusion. A Beckman Coulter Static Light Scattering (SLS) Particle Analyzer (LS13-320) was used to determine the particle size distributions (PSDs) of the OPC, the PCM microcapsules, and the quartz sand after ultrasonication in isopropanol to ensure dispersion of inclusions to primary particles (Figure 1). The complex refractive indices of these materials at a wavelength of $750 \mathrm{~nm}$ were taken as 1.70 $+0.10 i[75], 1.53+0.00 i[76]$, and $1.54+0.00 i$ [77], respectively. The maximum uncertainty in the PSDs was $\approx 6 \%$ based on 6 replicates.

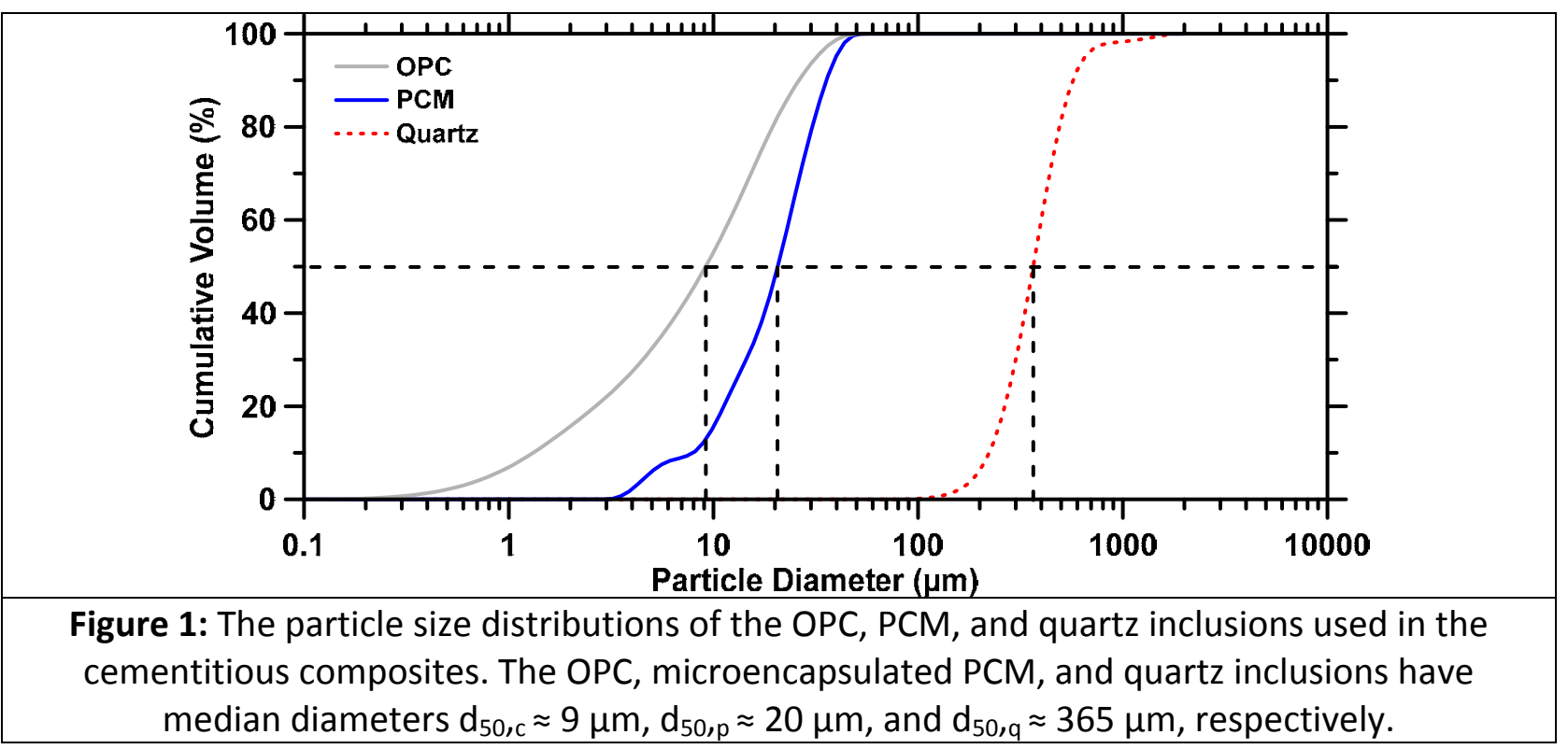

Model cementitious composites were prepared as per ASTM C305 [78] in the form of cement pastes (OPC + water mixture), PCM mortars (PCM + cement paste), quartz mortars (quartz + cement paste) and mixed mortars (PCM + quartz + cement paste). All mixtures were prepared at a fixed water-to-cement ratio $\mathrm{w} / \mathrm{c}=0.45$ on a per mass basis. Inclusions were added at 
volume fractions of $0.05,0.10,0.20$, and 0.30 for PCM mortars and at $0.10,0.20,0.30$, and 0.55 for quartz mortars. Two series of mixed mortars, i.e., those containing both PCM and quartz inclusions, were also evaluated for total inclusion volume fractions of $0.30(0.10,0.15,0.20$ PCM; remainder quartz) and 0.55 (0.10, 0.20 PCM; remainder quartz), respectively. Glenium $7500^{\circledR}$, a high range water-reducing admixture was used to enhance the fluidity of the mixtures. The dosage of water-reducer used corresponds to a maximum level of $2 \%$ by mass of cement.

The modulus of elasticity was measured for all mixtures as described in ASTM C469 [79] - a standard procedure for measuring such properties using cylindrical specimens (diameter $x$ height, $10.16 \mathrm{~cm} \times 20.32 \mathrm{~cm}$ ). Measurements were carried out after 1, 3, 7, and 28 days for specimens cured at $25.0 \pm 0.1{ }^{\circ} \mathrm{C}$ in saturated limewater (i.e., under immersion). Modulus of elasticity was measured using a MTS 311.31 closed-loop servo-hydraulic instrument provisioned with digital data acquisition and recording facilities. Quick-setting gypsum plaster "Hydrostone" was used for capping the cylindrical specimens. This ensured that the specimen was aligned with the loading axis and that the ends were in proper contact with the compression platens. The experimental chord modulus of elasticity $E_{\text {eff }}$ (i.e., equivalent to $E_{m}$ in the case of plain cement paste) of the composite specimens was calculated according to ASTM C469 [79]

$$
E_{\text {eff }}=\frac{\sigma_{2}-\sigma_{1}}{\varepsilon_{2}-\varepsilon_{1}}
$$

where $\sigma_{2}$ is the stress developed at $40 \%$ of the peak load, $\sigma_{1}$ is the stress developed at strain $\varepsilon_{1}$ $=50 \mu \varepsilon$, and $\varepsilon_{2}$ is the strain produced by stress $\sigma_{2}$. These parameters are illustrated in Figure 2 . The data reported represent the average of three replicates, with a coefficient of variation of $\approx$ $7 \%$.

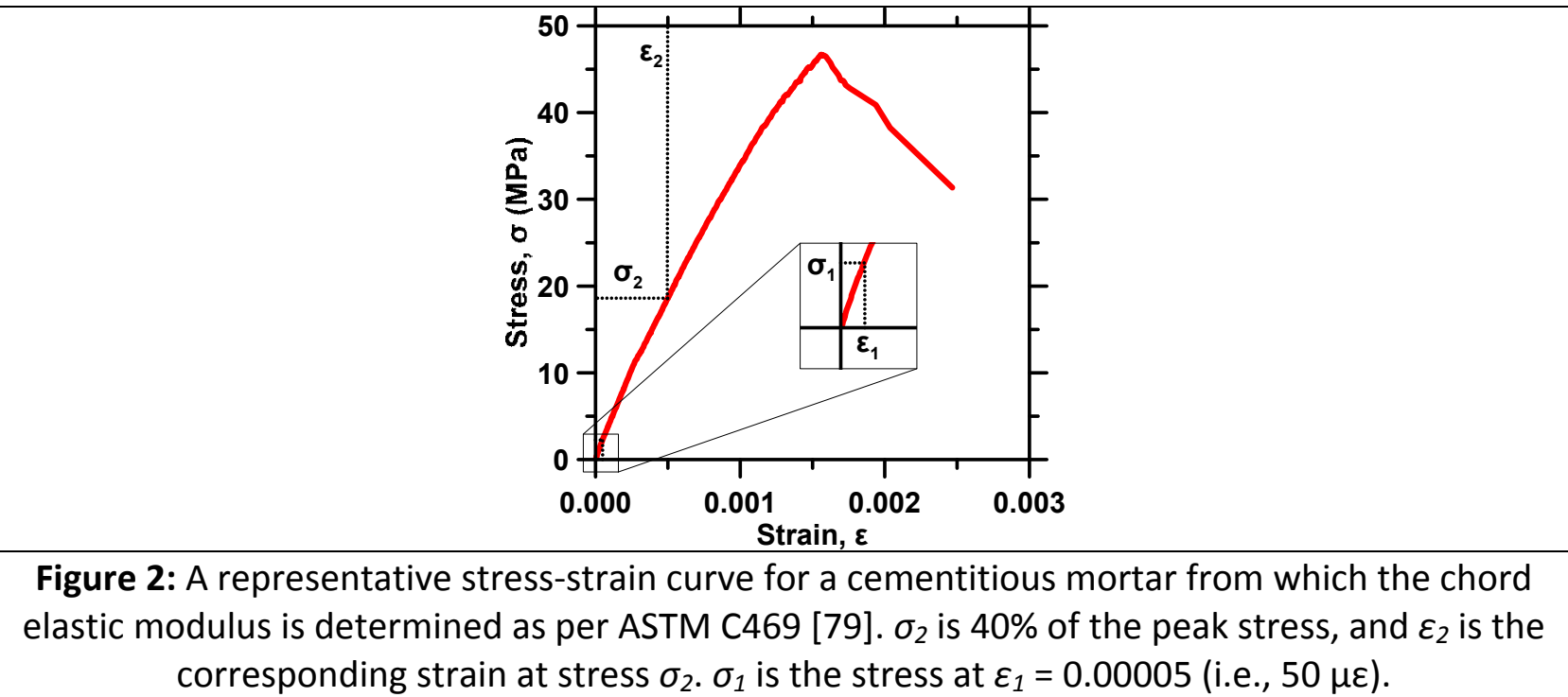


316 It should be noted that the selection of $40 \%$ of peak load is made to ensure compliance to 317 ASTM C469, as this stress level often corresponds to service-stress levels in concrete. To 318 investigate the validity of the assumption of linear elasticity in this stress regime, the modulus 319 of elasticity was calculated using stresses in the range $10 \% \leq \sigma_{2} \leq 40 \%$. Figure 3 indicates that the modulus of elasticity of single inclusion mortars is nearly independent (within experimental error) of stress level, suggesting modest damage accumulation in this stress range, as is desirable to ensure a nearly linear elastic response [80]. While the modulus of elasticity begins to increase for stress levels lower than $10 \%$, this is expected, as the measured chord modulus of elasticity begins to approach a value similar to the dynamic elastic modulus of the composite $[2,81]$. The influence of the upper stress level on the modulus of elasticity data is negligible in all cases excluding those representing the highest inclusion loadings (i.e., $\phi_{\mathrm{q}}=0.55$, and $\phi_{\mathrm{p}}=$ 0.30 ), which demonstrate an increased tendency for microcracking-induced softening due to their larger ITZ volume fraction. These aspects and their potential influences on the EMA predictions are discussed in detail in the supplementary information (Section S2). The data throughout the present study is calculated in accordance to ASTM C469, unless otherwise noted.

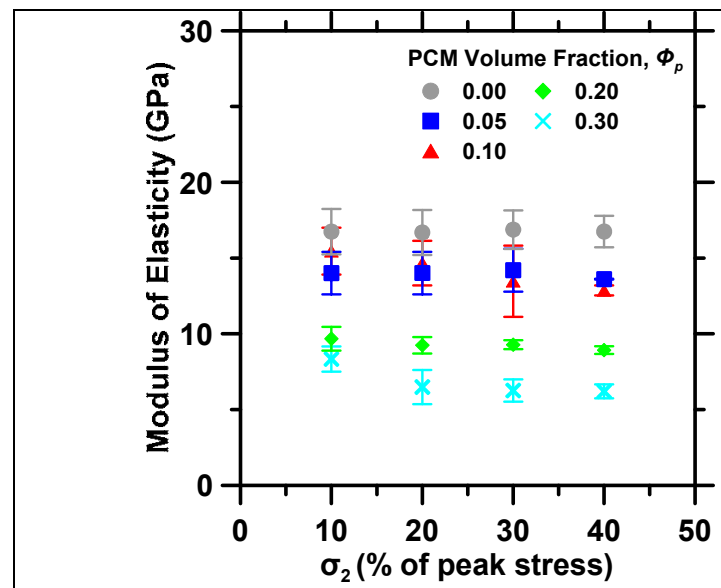

(a)

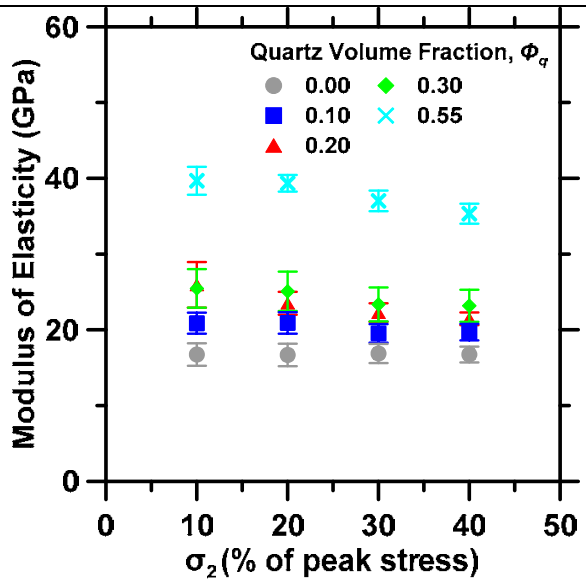

(b)

Figure 3: The influence of the upper stress level used to calculate the modulus of elasticity of:

(a) PCM mortars and (b) quartz mortars. The calculated modulus of elasticity shows little dependence on $\sigma_{2}$ (within measurement error).

333

Measurements of compressive strength of the composites were carried out as per ASTM C109 [82] using cubic specimens $(5 \mathrm{~cm} \times 5 \mathrm{~cm} \times 5 \mathrm{~cm}$ ) after curing for 1, 3, 7, and 28 days at $25.0 \pm 0.1$ ${ }^{\circ} \mathrm{C}$ in saturated limewater. The strength reported is the average of three replicates samples with a coefficient of variation of $\approx 5 \%$.

The modulus of elasticity and Poisson's ratios of the microencapsulated PCM (subscript " $p^{\text {") }}$ and quartz (subscript " $q$ ") inclusions were taken from the literature as: $E_{p}=0.0557 \mathrm{GPa}, v_{p}=$ 
3410.499 [83], and $E_{q}=72 \mathrm{GPa}, v_{q}=0.22$ [84]. The measured modulus of elasticity of the plain

342 paste was used as the age-dependent modulus of the matrix, which at 28 days was $E_{m}=16.75 \pm$ $3431.09 \mathrm{GPa}$ The Poisson's ratio of the cement paste matrix was selected as $v_{m}=0.22$ [2]. Unless 344 stated, the results presented represent the modulus of elasticity and compressive strength of 345 the composite specimens at an age of 28 days. This is because structural design codes (e.g., $\mathrm{ACl}$ 346318 [85]) often consider material properties at 28 days as design parameters.

\section{Results and Discussion}

\subsection{Experimental Data}

350 Figure 4 shows the modulus of elasticity and compressive strength measured on a series of 351 model composites containing microencapsulated PCM inclusions (i.e., PCM mortars), quartz 352 inclusions (i.e., quartz mortars) and PCM + quartz inclusions (i.e., mixed mortars). As expected, 353 increasing volume fractions of inclusions less stiff than the cement paste matrix decreased the 354 composite stiffness [20], while increasing stiffer inclusion volumes fractions increased 355 composite stiffness $[33,37,40,86]$. More interestingly, the reduction in modulus of elasticity due to the addition of soft inclusions was somewhat mitigated by the simultaneous addition of stiff inclusions. This is significant as it demonstrates a method for reducing the stiffness reduction noted in cementitious composites due to microencapsulated PCMs or other soft inclusion additions. Thus, for particular combinations of inclusion volume fractions, the composite stiffness can be greater than that of the paste matrix. This assures the existence of a critical ratio of stiff-to-soft inclusions such that composite stiffness is equivalent to that of the paste.

The compressive strength of single inclusion mixtures showed an upper limit that was enforced by the strength of the paste matrix in the case of quartz mortars, and a lower limit that scaled in proportion to the PCM inclusion volume fraction. This is expected as the paste is the weakest-link in the quartz mortar, but when present, PCM microcapsules become the critical strength controlling defects whose influence increases with their content in the system. It should be noted that the introduction of PCM microcapsules has no appreciable effect on the hydration of cement paste (see Fernandes et al. [9]). Therefore, the cement paste matrix is expected to demonstrate equivalent properties, regardless of PCM content. 


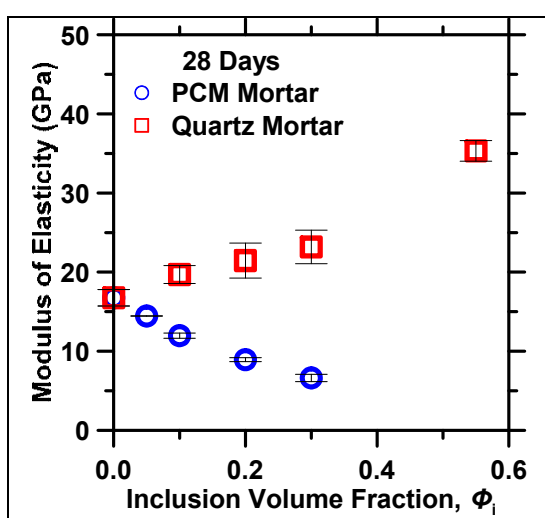

(a)

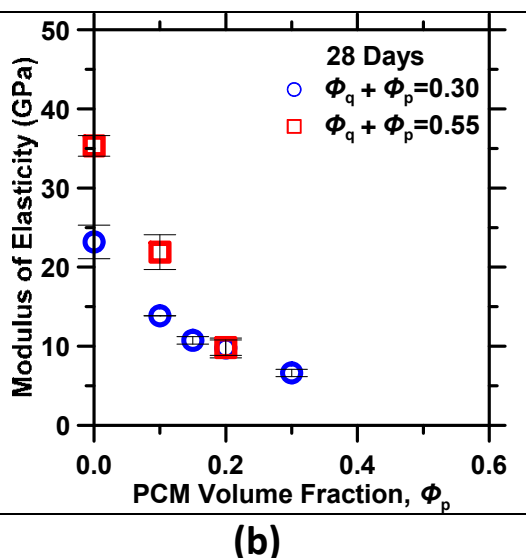

(b)

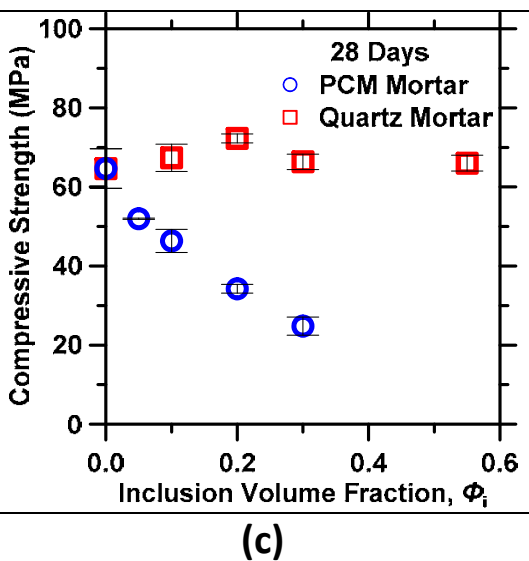

(c)

Figure 4: The experimentally measured modulus of elasticity for: (a) single inclusion mortars, and (b) mixed mortars; and (c) the compressive strength of single inclusion mortars.

372

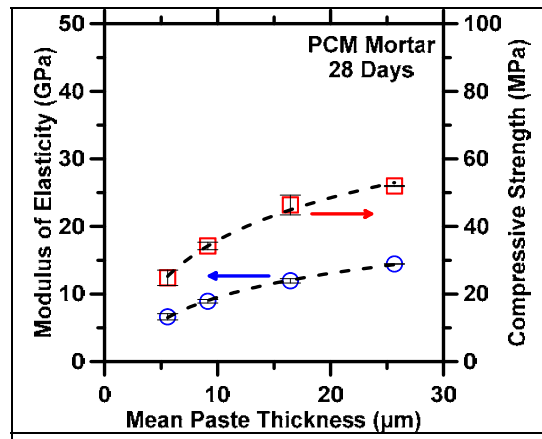

(a)

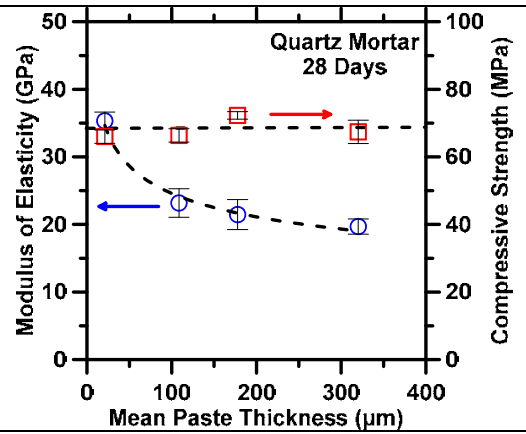

(b)

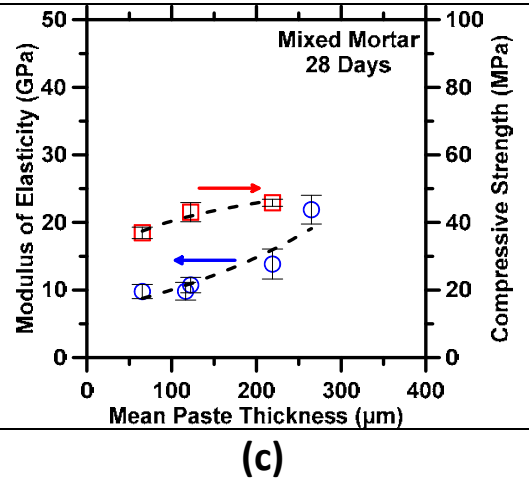

(c)

Figure 5: Correlations between measured mechanical properties and calculated mean paste thickness between inclusions for: (a) PCM mortar, (b) quartz mortar, and (c) mixed mortars. 
389 The measured modulus of elasticity and the compressive strength of each class of composite was investigated as a function of the mean spacing between inclusion surfaces, which approximates the thickness of the paste matrix (i.e., including pores and the ITZ) between inclusions [93]. This parameter is qualitatively, and in 1D, similar to the MSA, as they both describe the "load-bearing skeleton" of the composite. Figures 5(a) and 5(b) plot compressive strength and modulus of elasticity as a function of mean paste thickness between inclusion surfaces for PCM mortars and quartz mortars, respectively. Note that the results presented do not consider the effects of inclusion agglomeration, and thus are more qualitative than exact. The modulus of elasticity of PCM mortars increased as inter-PCM microcapsule distance increased due to the increasing thickness of the solid paste. The opposite trend was observed in quartz mortars, which are in essence an inverted system (stiff inclusions separated by a somewhat more compliant matrix). These mean distances were correlated to the volume fraction of inclusions in each composite. As an example, an increase in the inclusion volume fraction from 0.10 to 0.30 reduced inter-PCM microcapsule spacing from 16.4 to $5.6 \mu \mathrm{m}$, and reduced inter-quartz particle spacing from 320.2 to $108.5 \mu \mathrm{m}$. This was a reflection of the small size of PCM inclusions relative to quartz inclusions, as many more PCM microcapsules than quartz particles were required to achieve a given volume fraction. Figure 5(c) illustrates the influence of the spacing between all inclusion particles (both stiff and soft) on modulus of elasticity in mixed mortars. Note that this interparticle distance was approximately equivalent to the inter-PCM microcapsule distance as the PCM microcapsules controlled the paste film thickness (and thus the material's failure response) due to their small size and weak character. The mixed mortars showed an increase in the modulus of elasticity with spacing, demonstrating that the thickness of the solid (cement paste + quartz) between PCM inclusions acted as the critical stiffness-controlling parameter as suggested above.

The compressive strength of the composites followed trends similar to the modulus of elasticity when PCM inclusions were present (Figures $5 \mathrm{a}$ and $5 \mathrm{c}$ ). As expected, the strength of PCM mortars decreased significantly with decreasing PCM spacing (increasing PCM volume fraction). In quartz mortars (Figure 5b), compressive strength was approximately independent of inclusion spacing and quartz content. Thus, quartz inclusions were unable to influence the compressive strength of the composite, as the weaker cement paste acted as the critical (failure inducing) link. As a result, in contrast to the modulus of elasticity, the compressive strength reduction due to PCM microcapsules addition in mixed mortars could not be offset by any supplemental stiff inclusion additions. Thus, in mixed mortars, the PCM microcapsule volume fraction controlled compressive strength regardless of quartz inclusions, as the PCMs acted as 


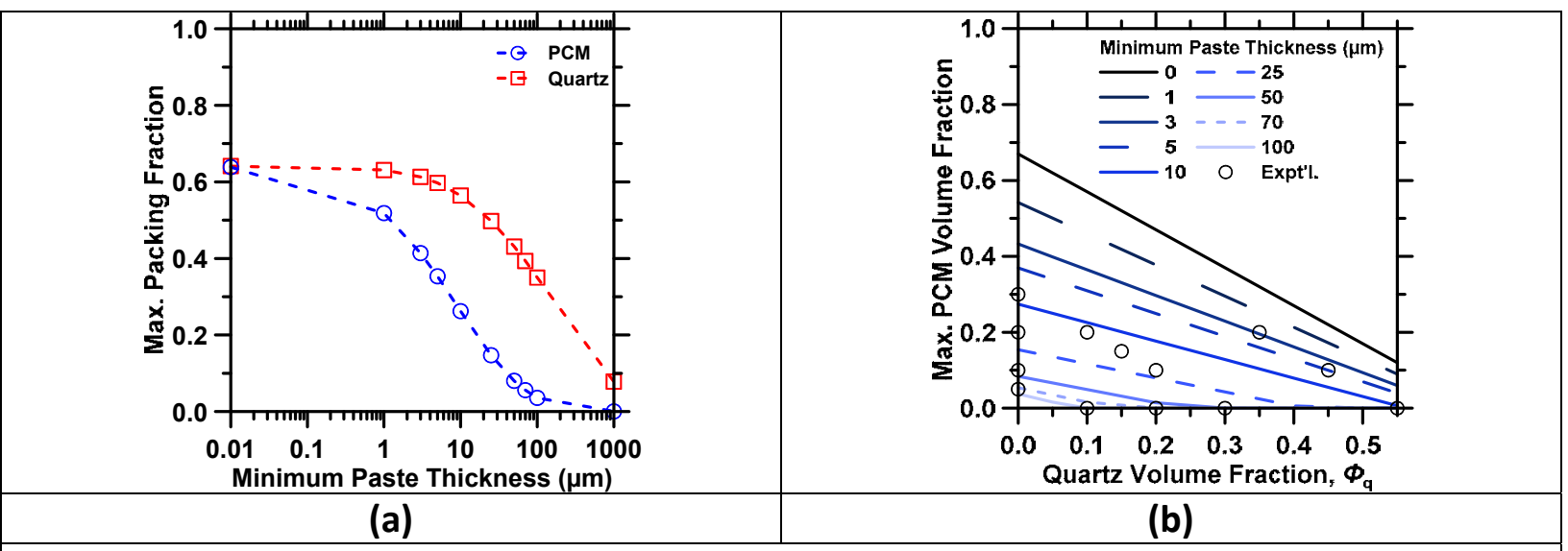

Figure 6: (a) The maximum packing limit (by volume) of inclusions in single-inclusion mortars as a function of minimum paste thickness between inclusions and (b) the maximum PCM loading as a function of the quartz volume fraction in mixed mortar systems. These calculations utilized the particle size distributions of each inclusion type displayed in Figure 1.

426

427

428

429

430

431

432

433

434

435

436

437

438

439

440

441

442

443

444

445

446

447

448

449

One practical limitation associated with embedment of PCMs into cementitious materials that is elucidated by the microstructure constructions is the inability to pack significant volume fractions of these particles into a composite while maintaining a continuous paste (matrix) layer between inclusions. For example, Figure 6(a) shows the maximum volumetric loading of inclusions for different assumed paste "film" thicknesses. As a point of validation of the packing algorithm, for random close packing of polydisperse particulates, the maximum inclusion packing fraction was $\approx 0.67$ as the minimum surface-to-surface distance approached zero. On account of their larger particle size, quartz particles may be included at higher volume fractions within the composite than PCM microcapsules while maintaining the same paste film thickness.

To aid in the design of cementitious mortars containing PCMs, the packing algorithm was used to calculate the largest volume fraction of PCM which can be embedded simultaneously with quartz (see Figure 6b). The compositions of the mixtures considered in this study are also included in this figure for the sake of comparison. The minimum inter-surface distance achieved in these mixtures was around $3 \mu \mathrm{m}$ for the mixture containing inclusion volume fractions of 0.20 PCM microcapsules and 0.35 quartz particles. Assuming that this minimum spacing of around $3 \mu \mathrm{m}$ was required to maintain a continuous matrix, the maximum permissible PCM microcapsule volume fraction (i.e., without quartz inclusions) that was achievable was $\phi_{\mathrm{p}} \approx 0.43$, which agreed qualitatively with our experimental observations. For example: PCM mortars produced for $\phi_{p}>0.40$ were non-cohesive highlighting that a minimum paste layer is needed to ensure cohesion.

\subsection{Single-Inclusion Mortars}




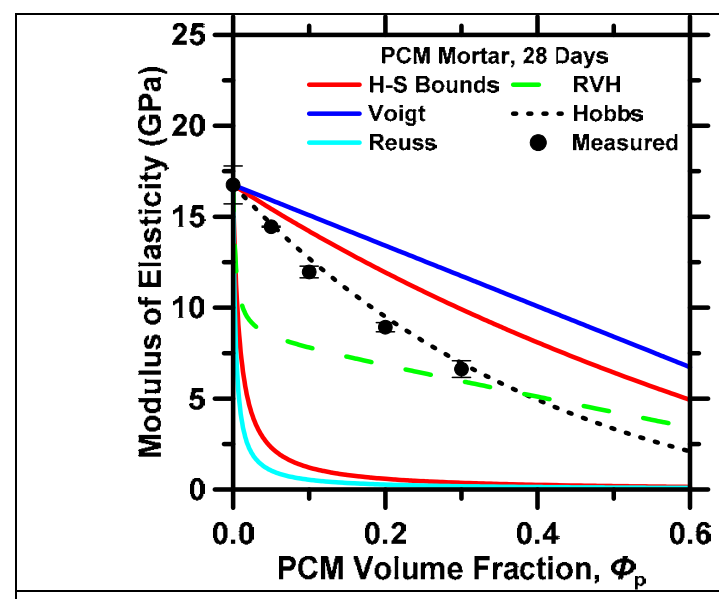

(a) volume fractions considered.

Figure 7 displays the modulus of elasticity predicted by the Hobbs EMA, the Reuss-Voigt (R-V) equations, and Hashin-Shtrikman (H-S) bounds for composites containing either PCM or quartz, respectively. As expected, the $\mathrm{H}-\mathrm{S}$ and $\mathrm{R}-\mathrm{V}$ bounds were unreasonably wide in the case of the PCM mortar, due to the substantial mismatch in matrix-inclusion moduli, i.e., since $170 \leq E_{m} / E_{p}$ $\leq 300$, for paste ages ranging from 1 day to 28 days. The Hobbs EMA, implemented for the case of substantial mismatch in the Poisson's ratio between the matrix and inclusions (Equation $2 b$ ), was able to favorably describe the effective moduli of PCM mortars across the entire range of

Figure 7: The predictions of two-component EMAs for: (a) PCM mortars and (b) quartz mortars compared with experimental data as a function of the inclusion volume fractions $\phi_{p}$ and $\phi_{q}$, respectively.

459

460

461

462

463

464

465

466

467

468

469

470

471

472

473

474

When applied to the quartz mortars (not shown), essentially all EMAs were able to describe the composite modulus favorably with all predictions lying within the narrow $\mathrm{H}-\mathrm{S}$ bounds. This was attributed to the similarity in properties, i.e., modulus of elasticity and Poisson's ratio, between quartz and the cement paste. Here, the ratio $E_{m} / E_{q}$ ranged from 0.13 to 0.23 for cement paste aging from 1 day to 28 days. Once again, the Hobbs EMA (see Equation 2a) favorably predicted composite moduli, in this case coinciding with the lower $\mathrm{H}-\mathrm{S}$ bound.

Next, the K-T, M-T, GSCM, and D-EMT EMAs were evaluated for their ability to predict the moduli of elasticity of composites. In the case of the PCM mortars, the majority of these EMAs produced predictions equivalent to, or in proximity to the H-S upper bound (Figure 8a), i.e., resulting in overestimations vis-à-vis the experimental data. One explanation for this may be that an essential microstructure component, such as the ITZ, was disregarded. To assess this possibility, an ITZ was introduced into the calculation scheme under the assumptions stated in

\section{Section 2.3.}




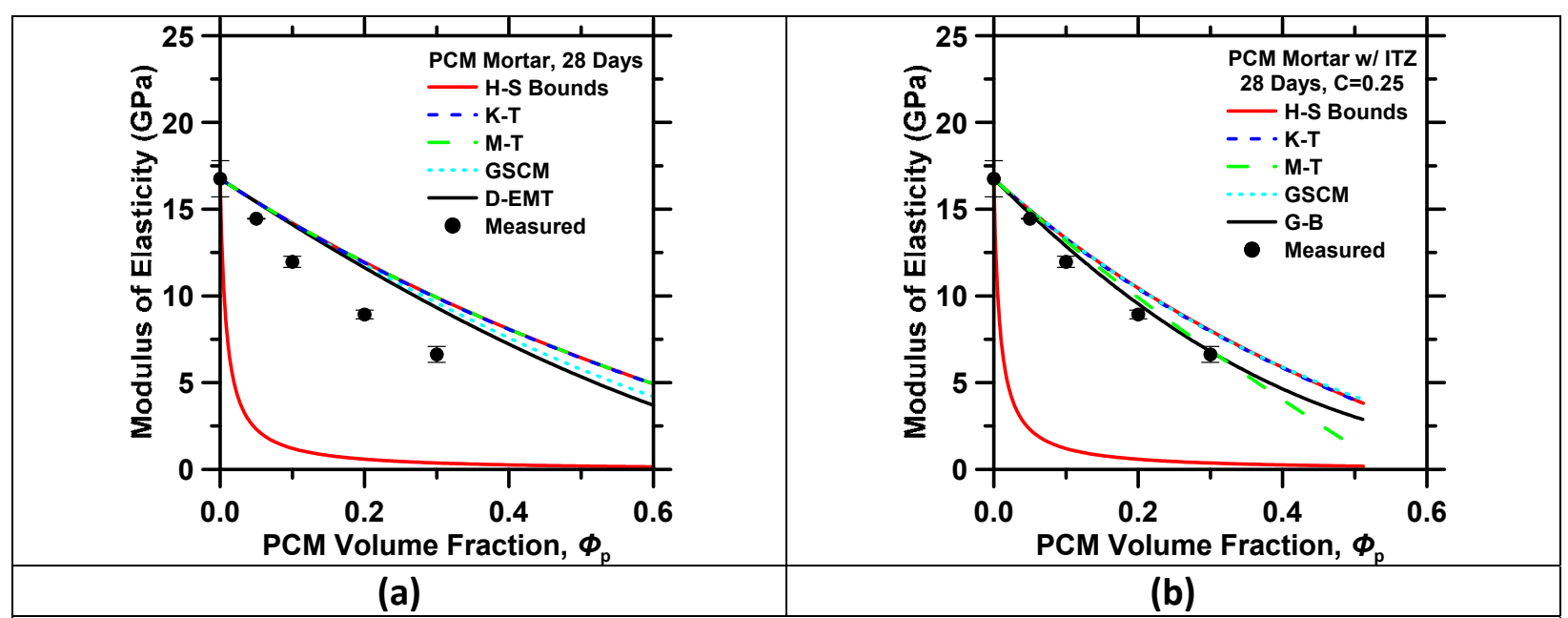

Figure 8: The predictions of: (a) two-component EMAs for PCM mortars and (b) threecomponent EMAs, which consider the presence of an ITZ in PCM mortars, as a function of the PCM volume fraction. The overestimation of the modulus of elasticity noted in Figure $8(a)$ is suitably corrected by the consideration of an ITZ component.

475

476

477

478

479

480

481

482

483

484

485

486

487

488

489

490

491

492

493

494

495

496

Figure $8(\mathrm{~b})$ indicates that EMA predictions improved considerably upon consideration of the ITZ, to more accurately describe experimental data. For example, the H-S upper bound reduced, while the lower H-S bound remained unchanged. The K-T EMA remained similar to the H-S upper bound while the M-T formulation significantly reduced, and showed a near-linear reduction in modulus of elasticity with the PCM volume fraction. The D-EMT method utilized by Garboczi and Berryman (G-B) [55] showed excellent agreement with the experimental data. The success of the G-B EMA was perhaps unsurprising as it was previously demonstrated to accurately predict the elastic response of cementitious materials containing an ITZ [55].

Following the example of PCM mortars, the same EMAs were also evaluated for quartz mortars with and without consideration of an ITZ component. In both two/three-component systems, the $\mathrm{H}$-S lower bound provided an acceptable prediction of the effective composite modulus. Given the small contrast in the matrix-inclusion-ITZ properties, the consideration of an ITZ, or lack thereof had little bearing on the results. It should be noted that two-component EMAs, in spite of their physical incompleteness (i.e., lack of an ITZ component) were sufficient because the ITZ exerted little effect on quartz mortars' properties. Similarly, three-component EMAs containing an ITZ were sufficient because the added detail (i.e., accounting for the ITZ phase), had little if any effect. This may suggest that in the case of inclusions similar to the paste matrix, at moderate volume fractions for moderately sized inclusions, the use of two-component EMAs is reasonable. 


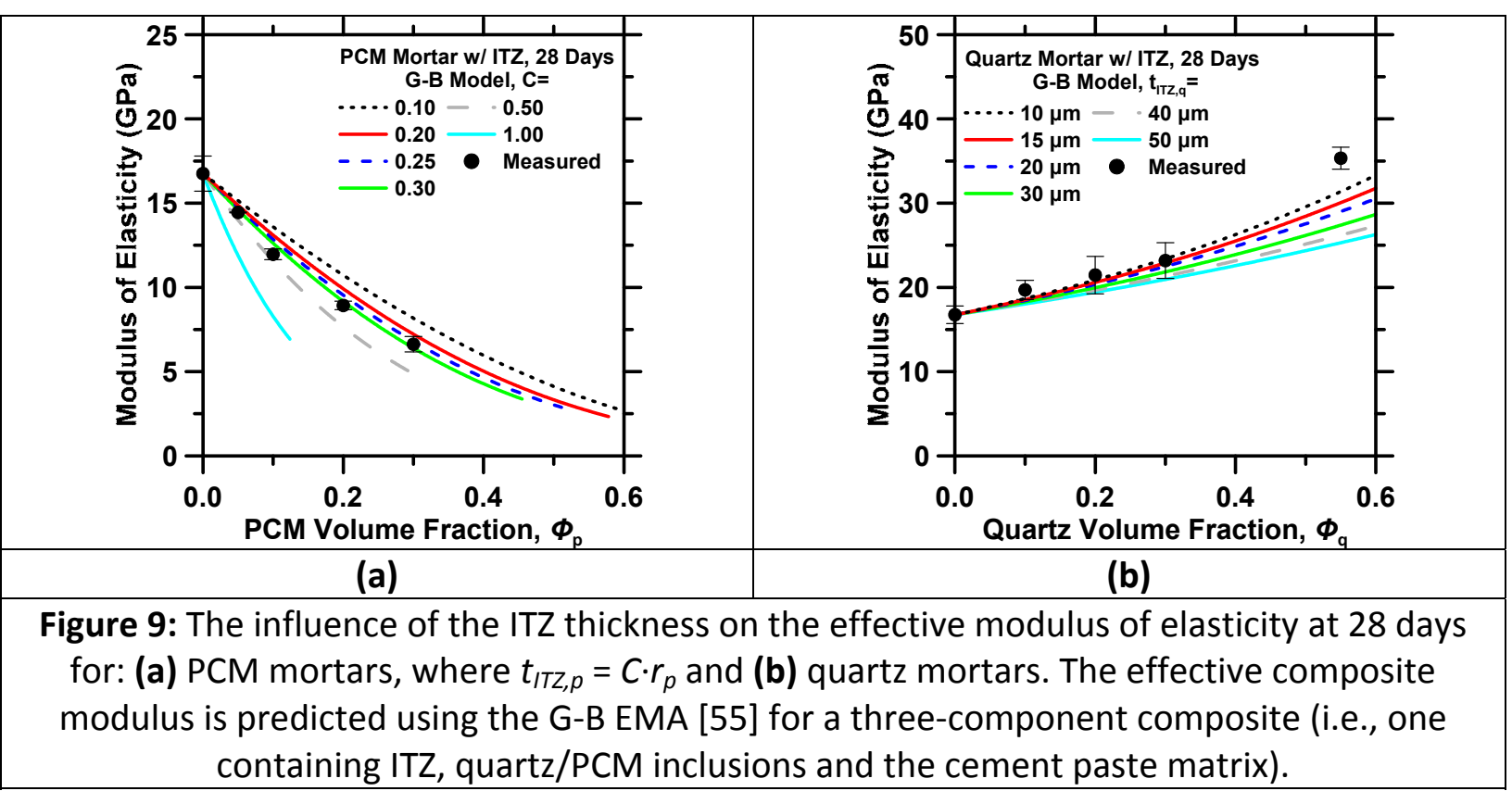

497

498

499

Since the selected ITZ characteristics impact the predictions of EMAs, the effect of the ITZ layer thickness (i.e., for a constant stiffness) was elucidated by parametric analysis carried out using the EMA of Garboczi and Berryman (G-B, Figure 9 and Figure S4 in supplementary information). A range of ITZ thickness from 0.1-to-1.0 times the median PCM microcapsule radius and from 10-to-50 $\mu \mathrm{m}$ around the quartz particles was considered [63,65,94,95]. At any given PCM volume fraction (e.g., $\left.\phi_{p}=0.30\right)$, the volume fraction of the ITZ $\left(\phi_{I T Z, p}\right)$ varied from 0.099 -to0.36 as $C$ varied from 0.10 to 0.30 . For the same volume fraction of quartz $\left(\phi_{q}\right)$, increasing the ITZ thickness from $10 \mu \mathrm{m}$ to $50 \mu \mathrm{m}$ increased the ITZ volume fraction $\left(\phi_{\text {ITZ,q }}\right)$ from 0.15 to 0.32 . When considering PCM mortars (Figure 9a), the ITZ volume fraction became very large at high inclusion loading. For example, when the volume fraction of microencapsulated PCM is $0.30, C$ $>0.43$ yielded a total volume fraction of ITZ and PCM microcapsules in excess of one. This was due to the lack of consideration of overlap of the ITZ shells. The error induced by neglecting ITZ overlap was minimized by selecting thin ITZ layers and considering composites with smaller inclusion loadings. Although the PCM modulus of elasticity was far lower than that of the ITZ, increasing the ITZ volume fraction significantly reduced the predicted modulus (see Figure 9a).

The G-B model predictions for quartz mortars showed a similar reduction in composite properties with increasing ITZ thickness (Figure 9b), although the volumetric dependence on ITZ was weaker. This was expected as the stiffness of the ITZ was the least of all components in the quartz mortar system. For the input properties selected herein, an effective ITZ thickness of 10 $\mu \mathrm{m}$ appeared appropriate in support of the choices made in Section 2.3 above.

\subsection{Mixed Inclusion Mortars}


521 In the next step, the effective moduli of the cementitious composites containing both stiff and

522 soft inclusions were modeled. Here, the EMA implementations considered discrete

523 components, including inclusions of quartz and microencapsulated PCM, the paste matrix, and

524 in certain cases, the ITZ. When the ITZ was not considered, only the EMAs of Hobbs and the

525 RVH average provided acceptable estimates across the range of formulations considered (see

526 Figure 10a-b). Note however, since some of the EMAs only consider two-component

527 composites, e.g., in Hobbs EMA, a two-step homogenization process was followed. Thus, first,

528 quartz particles were embedded into the cement paste matrix (using Equation 2a), which

529 yielded an effective matrix into which the PCM microcapsules were then embedded using

530 Equation 2(b). Further discussion is noted in Section S.2 in the supplementary information.

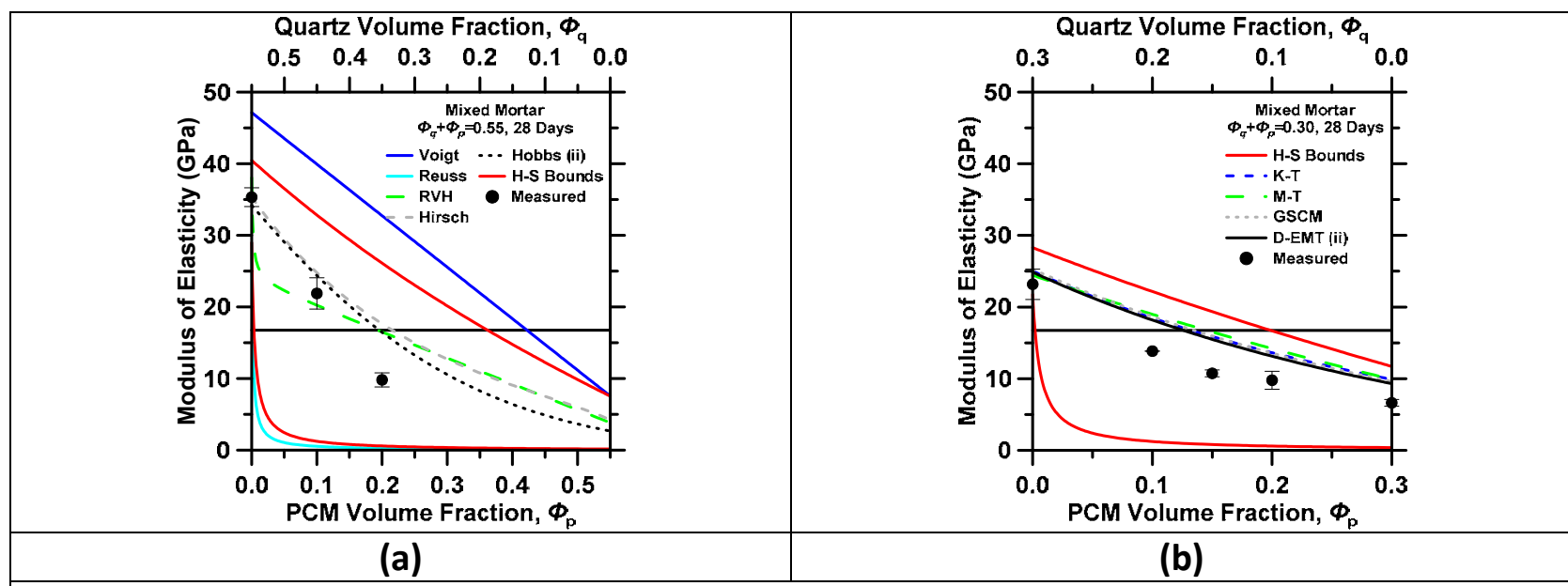

Figure 10: The measured and calculated modulus of elasticity for composites containing both soft and stiff inclusions for: (a) 0.55 inclusion volume fraction, and (b) 0.30 inclusion volume fraction. The solid horizontal line shows the modulus of elasticity of the plain cement paste matrix. EMAs denoted (ii) represent quartz-first homogenization (see SI).

532

533

534

535

536

537

538

539

540

The effective composite modulus for both mixed mortars was generally overestimated, although the D-EMT and K-T EMAs provided the most favorable results. In the case of multicomponent composites (i.e., those having more than 2-inclusion components), the order of homogenization of the inclusions into the paste matrix influenced the predicted moduli. This order-dependence of inclusion homogenization in the D-EMT has been previously discussed [56]. A clear comparison of these predictions is provided in the supplementary material (Figure S3). 


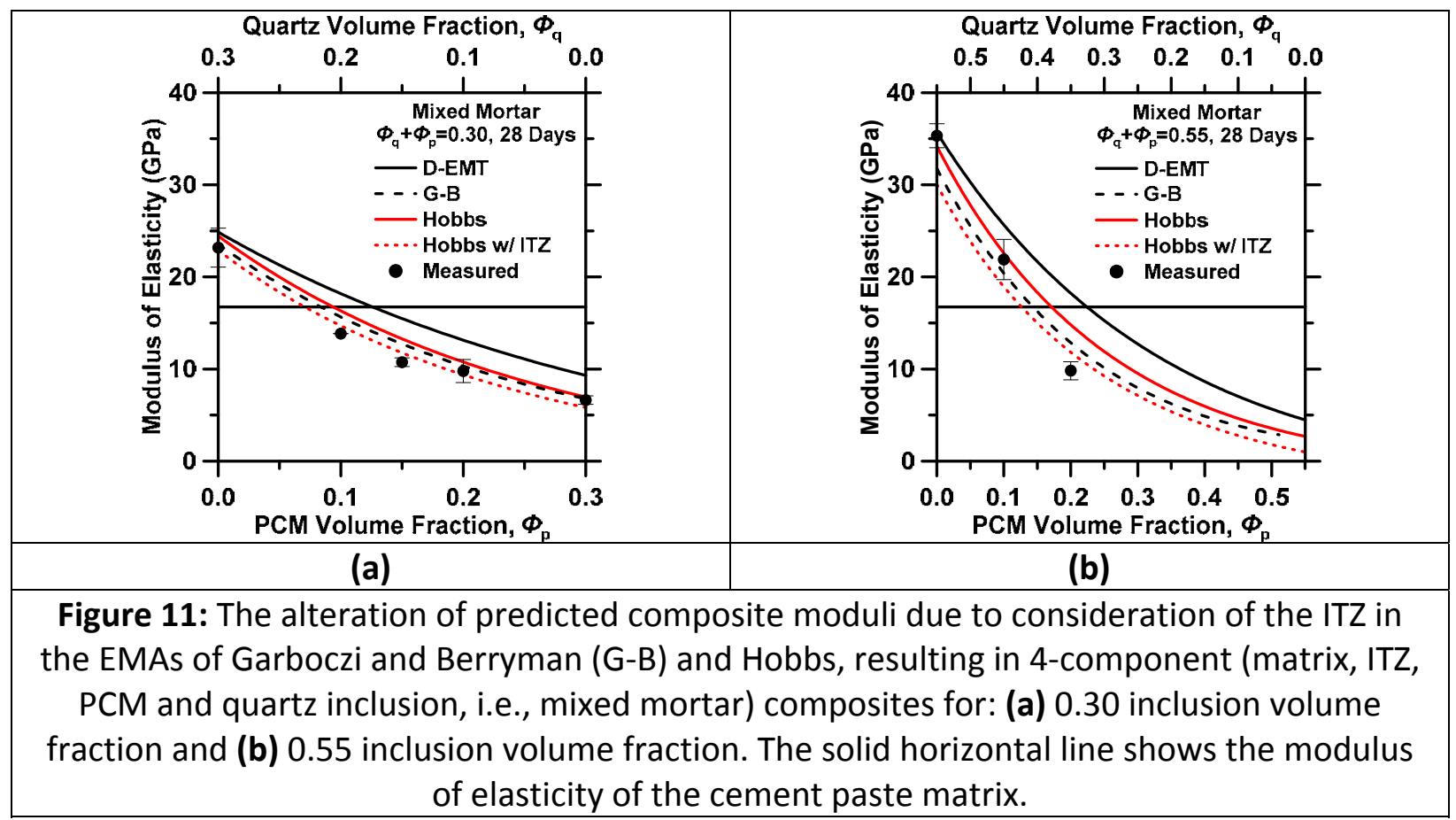

541

542

543

544

545

546

547

548

549

550

551

552

553

554

555

556

557

558

559

560

561

562

Since the ITZ was observed to impact EMA predictions (Figure 8), this component was added to composites containing both the soft (PCM microcapsules) and stiff (quartz particles) inclusions. Based upon the modulus of elasticity estimations shown in Figure 10, the EMAs of Hobbs and of Garboczi and Berryman (G-B) were selected for predicting the composite moduli (see also Figure S3 in the supplementary information). For both EMAs, a sequential implementation was carried out such that each inclusion was first homogenized with its surrounding ITZ, and then introduced into the matrix. In each EMA, it was observed that consideration of the ITZ reduced the predicted effective modulus of the composite, with a stronger effect as PCM volume fraction increased (see Figure 11). The fit of the EMAs to experimental data was improved when the ITZ was considered (with $t_{I T Z, q}=10 \mu \mathrm{m}$ and $C=0.25$ ).

\subsection{Design Rules for Ensuring Performance Equivalence of Stiffness in Cementing Composites}

Cementitious composites containing both PCM and quartz inclusions have demonstrated elastic moduli greater than or less than that of the paste matrix, depending on the volume fraction of each type of inclusion in the composite. This suggested that strategic additions of stiff and soft inclusions may be used to ensure "no compromise" (i.e., vis-à-vis, the cement paste matrix) in the modulus of elasticity of cementitious materials containing PCMs. For design purposes, such equivalence in properties can be achieved by identifying the critical ratio of inclusion volume fractions $\left(\phi_{q} / \phi_{p}\right)$ such that the composite modulus of elasticity remains:
(a) similar to the paste matrix, i.e., $E_{\text {eff }} / E_{m}=1$, or,

(b) superior to the paste matrix, i.e., $E_{e f f} / E_{m}>1$. 
563 As an example, this critical ratio was calculated using the Hobbs EMA [39] on account of its 564 simple implementation and its ability to accurately predict composite properties.

565

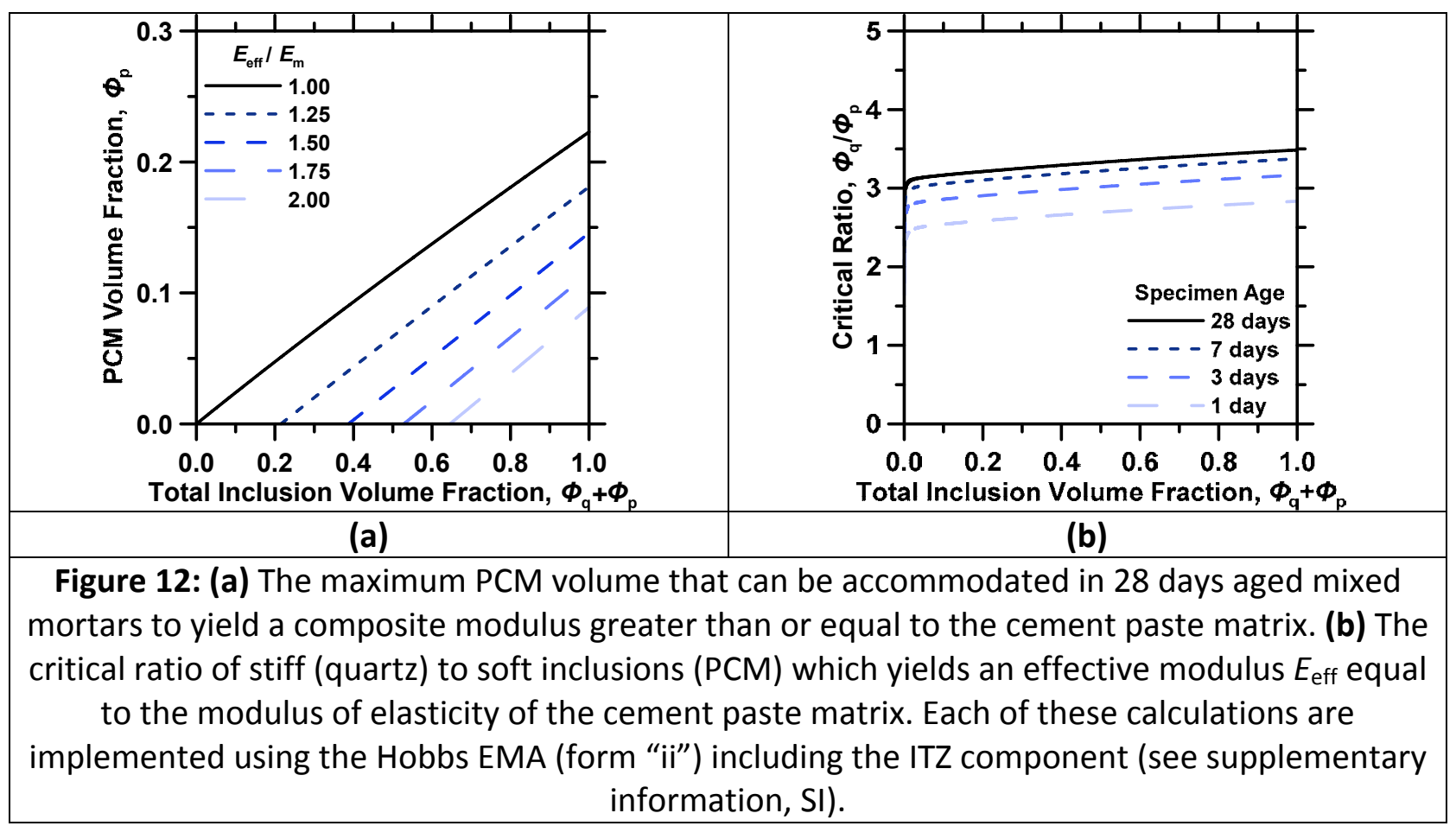

566

567

568

569

570

571

572

573

574

575

576

577

578

579

580

581

582

583

584

Conditions (a) and (b) were sequentially input into the Hobbs (i.e., four-component; PCM, quartz, cement paste matrix, and ITZ) EMA, which was solved numerically for $\phi_{p}$ to yield a given (total) inclusion volume fraction $\phi_{q}+\phi_{p}$. These values of PCM volume fraction are displayed in Figure 12(a) for composite elastic moduli ranging from 1.0 to 2.0 times the cement paste at 28 days age. In general, to attain a higher modulus, or to accommodate a higher PCM volume, the quantity of quartz or any other inclusions stiffer than the matrix, needs to be increased to counteract the softening caused by a given PCM dosage. Note, however, that below a certain total inclusion volume, PCM may not be introduced into the composite, since the quantity of quartz present is far too low to produce the desired modulus of elasticity.

Dividing the quartz volume by the PCM volume yielded the critical ratio of the quartz-to-PCM inclusion volumes, as shown in Figure 12(b) as a function of the total inclusion volume in the composite. These results indicated that, in general, quartz inclusions should be present at a level $\approx 2.5$-3.5 times (i.e., due to age dependence) greater than the PCM volume fraction to produce a composite with a modulus of elasticity equivalent to that of the cement paste. The increase in critical ratio with volume fraction suggests that the PCM-associated ITZ played a significant role in decreasing the composite stiffness. The results were also strongly dependent on the modulus of the matrix (aging of matrix), the modulus of ITZ, and the volume of ITZ 
assumed. Embedding inclusion particles of even higher stiffness than quartz, or adding supplementary cementitious materials (e.g., silica fume $[2,27,96]$ ) to reduce the porosity of the ITZ may be additional means to reduce the critical ratio for maintaining mechanical performance. Note however, that the present study considered only the case of fine aggregate inclusions. As such, while it captured the effects of aggregate volume fraction and properties other aspects which improve mechanical load capacity in the case of coarse aggregates, e.g., that of aggregate frictional interlock and stress transfer, were not considered. Therefore, while the outcomes of this work serve as a design tool for ensuring property equivalence for cementitious composites containing PCMs, the designs rendered are expected to be somewhat conservative when extended to concretes [9].

\section{Summary and Conclusions}

The use of soft PCM microcapsules in functional cementitious materials requires estimations of their deleterious effects on the effective mechanical properties of the composite. To enable such estimations, the modulus of elasticity was measured for cement mortar formulations containing PCM microcapsules, quartz sand, or mixtures of both inclusions. A range of effective medium approximations (EMAs) were evaluated for their ability to predict the modulus of elasticity of such composites. Several EMAs were able to describe the stiffness of composites containing hard inclusions, which in this case had a small matrix-to-inclusion property mismatch. However, only the EMAs of Hobbs and of Garboczi and Berryman (G-B) were able to accurately describe the effective moduli of elasticity of PCM mortars and mixed (i.e., PCM + quartz) mortars across a wide range of inclusion volume fractions (up to 0.55). In each case, the accuracy of the EMAs was improved by considering the interfacial transition zone (ITZ) formed around the inclusion due to improper "packing" at the matrix-inclusion interface. A critical ratio of stiff/soft inclusion volume fractions was proposed as a design tool to formulate stiffnessequivalent cementing composites containing PCMs. The identification of more accurate EMAs offers structural engineers/designers a means to estimate the influence of PCMs, and other soft inclusions, on the modulus of elasticity of multifunctional concretes for construction applications.

\section{Acknowledgements}

The authors acknowledge financial support for this research provisioned by the California Energy Commission (Contract: PIR: 12-032), the University of California, Los Angeles (UCLA) and National Science Foundation (CMMI: 1130028) and an Infravation ERA-NET Plus Grant (31109806.0001). The contents of this paper reflect the views and opinions of the authors, who are responsible for the accuracy of the datasets presented herein, and do not reflect the views of the funding agency, nor do the contents constitute a specification, a standard or a regulation. This research was conducted in the Laboratory for the Chemistry of Construction Materials (LC ${ }^{2}$ ) 
662

663

664

665

666

667

668

and the core-facility Molecular Instrumentation Center (MIC) at the University of California, Los Angeles. As such, the authors acknowledge the support of these laboratories in making this research possible. The last author acknowledges discretionary support provided by the Edward K. and Linda L. Rice Endowed Chair in Materials Science.

\section{References}

[1] H.F.W. Taylor, Cement Chemistry, Thomas Telford, 1997.

[2] S. Mindess, J.F. Young, D. Darwin, Concrete, 2nd ed., Prentice Hall, Upper Saddle River, NJ, 2003.

[3] L.F. Cabeza, C. Castellón, M. Nogués, M. Medrano, R. Leppers, O. Zubillaga, Use of microencapsulated PCM in concrete walls for energy savings, Energy Build. 39 (2007) 113-119. doi:10.1016/j.enbuild.2006.03.030.

[4] P. Arce, C. Castellón, A. Castell, L.F. Cabeza, Use of microencapsulated PCM in buildings and the effect of adding awnings, Energy Build. 44 (2012) 88-93. doi:10.1016/j.enbuild.2011.10.028.

[5] V.V. Tyagi, D. Buddhi, PCM thermal storage in buildings: A state of art, Renew. Sustain. Energy Rev. 11 (2007) 1146-1166. doi:10.1016/j.rser.2005.10.002.

[6] A.M. Khudhair, M.M. Farid, A review on energy conservation in building applications with thermal storage by latent heat using phase change materials, Energy Convers. Manag. 45 (2004) 263-275. doi:10.1016/S0196-8904(03)00131-6.

[7] F. Kuznik, D. David, K. Johannes, J.-J. Roux, A review on phase change materials integrated in building walls, Renew. Sustain. Energy Rev. 15 (2011) 379-391. doi:10.1016/j.rser.2010.08.019.

[8] V.V. Tyagi, S.C. Kaushik, S.K. Tyagi, T. Akiyama, Development of phase change materials based microencapsulated technology for buildings: A review, Renew. Sustain. Energy Rev. 15 (2011) 1373-1391. doi:10.1016/j.rser.2010.10.006.

[9] F. Fernandes, S. Manari, M. Aguayo, K. Santos, T. Oey, Z. Wei, et al., On the feasibility of using phase change materials (PCMs) to mitigate thermal cracking in cementitious materials, Cem. Concr. Compos. 51 (2014) 14-26. doi:10.1016/j.cemconcomp.2014.03.003.

[10] M. Hunger, A.G. Entrop, I. Mandilaras, H.J.H. Brouwers, M. Founti, The behavior of self-compacting concrete containing micro-encapsulated phase change materials, Cem. Concr. Compos. 31 (2009) 731-743. doi:10.1016/j.cemconcomp.2009.08.002.

[11] M. Fenollera, J. Míguez, I. Goicoechea, J. Lorenzo, M. Ángel Álvarez, The influence of phase change materials on the properties of self-compacting concrete, Materials. 6 (2013) 3530-3546. doi:10.3390/ma6083530.

[12] P. Meshgin, Y. Xi, Y. Li, Utilization of phase change materials and rubber particles to improve thermal and mechanical properties of mortar, Constr. Build. Mater. 28 (2012) 713-721. doi:10.1016/j.conbuildmat.2011.10.039.

[13] N. Eldin, A. Senouci, Rubber-tire particles as concrete aggregate, J. Mater. Civ. Eng. 5 (1993) 478496.

[14] R. Siddique, T.R. Naik, Properties of concrete containing scrap-tire rubber-an overview., Waste Manag. 24 (2004) 563-9. doi:10.1016/j.wasman.2004.01.006.

[15] R. Siddique, J. Khatib, I. Kaur, Use of recycled plastic in concrete: a review., Waste Manag. 28 (2008) 1835-52.

[16] K.G. Babu, D.S. Babu, Behaviour of lightweight expanded polystyrene concrete containing silica fume, Cem. Concr. Res. 33 (2003) 755-762. doi:10.1016/S0008-8846(02)01055-4.

[17] D. Bouvard, J.M. Chaix, R. Dendievel, A. Fazekas, J.M. Létang, G. Peix, et al., Characterization and simulation of microstructure and properties of EPS lightweight concrete, Cem. Concr. Res. 37 (2007) 1666-1673. doi:10.1016/j.cemconres.2007.08.028. 
715

[18] H. Mazaheripour, S. Ghanbarpour, S.H. Mirmoradi, I. Hosseinpour, The effect of polypropylene fibers on the properties of fresh and hardened lightweight self-compacting concrete, Constr. Build. Mater. 25 (2011) 351-358. doi:10.1016/j.conbuildmat.2010.06.018.

[19] K. Miled, K. Sab, R. Le Roy, Particle size effect on EPS lightweight concrete compressive strength: Experimental investigation and modelling, Mech. Mater. 39 (2007) 222-240. doi:10.1016/j.mechmat.2006.05.008.

[20] K.J. Shin, B. Bucher, J. Weiss, Role of Lightweight Synthetic Particles on the Restrained Shrinkage Cracking Behavior of Mortar, J. Mater. Civ. Eng. 23 (2011) 597-605. doi:10.1061/(ASCE)MT.19435533.0000213.

[21] S. Popovics, A hypothesis concerning the effects of macro-porosity on mechanical properties of concrete, in: Fract. Concr. Rock, Springer, 1989: pp. 170-174.

[22] R. Le Roy, E. Parant, C. Boulay, Taking into account the inclusions' size in lightweight concrete compressive strength prediction, Cem. Concr. Res. 35 (2005) 770-775. doi:10.1016/j.cemconres.2004.06.002.

[23] H. Cui, W. Liao, S.A. Memon, B. Dong, W. Tang, Thermophysical and Mechanical Properties of Hardened Cement Paste with Microencapsulated Phase Change Materials for Energy Storage, Materials. 7 (2014) 8070-8087. doi:10.3390/ma7128070.

[24] A.M. Ghaly, M.S. Gill, Compression and Deformation Performance of Concrete Containing Postconsumer Plastics, J. Mater. Civ. Eng. 16 (2004) 289-296. doi:10.1061/(ASCE)08991561(2004)16:4(289).

[25] E. Ganjian, M. Khorami, A.A. Maghsoudi, Scrap-tyre-rubber replacement for aggregate and filler in concrete, Constr. Build. Mater. 23 (2009) 1828-1836. doi:10.1016/j.conbuildmat.2008.09.020.

[26] J. Xu, H. Chu, Y. Xu, Y. Li, L. Jiang, Prediction of compressive strength and elastic modulus of expanded polystyrene lightweight concrete, Mag. Concr. Res. 67 (2015) 954-962. doi:10.1680/macr.14.00375.

[27] K.L. Scrivener, A.K. Crumbie, P. Laugesen, The Interfacial Transition Zone (ITZ) Between Cement Paste and Aggregate in Concrete, Interface Sci. 12 (2004) 411-421. doi:10.1023/B:INTS.0000042339.92990.4c.

[28] P. Monteiro, J.C. Maso, J.P. Ollivier, The aggregate-mortar interface, Cem. Concr. Res. 15 (1985) 953-958.

[29] X. Ping, J.J. Beaudoin, R. Brousseau, Effect of aggregate size on transition zone properties at the portland cement paste interface, Cem. Concr. Res. 21 (1991) 999-1005. doi:10.1016/00088846(91)90059-Q.

[30] K. Maekawa, T. Ishida, T. Kishi, Multi-Scale Modeling of Structural Concrete, CRC Press, 2008.

[31] W. Voigt, Ueber die beziehung zwischen den beiden elasticitätsconstanten isotroper körper, Ann. Phys. (1889) 573-587.

[32] A. Reuss, Berechnung der fließ grenze von mischkristallen auf grund der plastizitätsbedingung für einkristalle, ZAMM - Z. Für Angew. Math. Mech. 9 (1929) 49-58. doi:10.1002/zamm.19290090104.

[33] T. Hansen, Influence of aggregate and voids on modulus of elasticity of concrete, cement mortar, and cement paste, ACl J. Proc. (1965) 193-216.

[34] R. Hill, The elastic behaviour of a crystalline aggregate, Proc. Phys. Soc. Sect. A. 65 (1952) 349-354.

[35] Z. Hashin, S. Shtrikman, A variational approach to the theory of the elastic behaviour of multiphase materials, J. Mech. Phys. Solids. 11 (1963) 127-140.

[36] L. Walpole, On bounds for the overall elastic moduli of inhomogeneous systems-I, J. Mech. Phys. Solids. 14 (1966).

[37] A.U. Nilsen, P. Monteiro, Concrete: a three phase material, Cem. Concr. Res. 23 (1993) 147-151.

[38] Z. Hashin, Analysis of composite materials-a survey, J. Appl. Mech. 50 (1983) 481-505. 
716

717

718

719

720

721

722

723

724

725

726

727

728

729

730

731

732

733

734

735

736

737

738

739

740

741

742

743

744

745

746

747

748

749

750

751

752

753

754

755

756

757

758

759

760

761

762

763

[39] D.W. Hobbs, The dependence of the bulk modulus, Young's modulus, creep, shrinkage and thermal expansion of concrete upon aggregate volume concentration, Matér. Constr. 4 (1971) 107-114. doi:10.1007/BF02473965.

[40] R. Zimmerman, M. King, P. Monteiro, The elastic moduli of mortar as a porous-granular material, Cem. Concr. Res. 16 (1986) 239-245.

[41] R. Christensen, K. Lo, Solutions for effective shear properties in three phase sphere and cylinder models, J. Mech. Phys. Solids. 27 (1979) 315-330.

[42] J.C. Smith, Simplification of van der Poel's formula for the shear modulus of a particulate composite, J. Res. Natl. Bur. Stand. Sect. Phys. Chem. 79A (1975) 419. doi:10.6028/jres.079A.007.

[43] R. Christensen, A critical evaluation for a class of micro-mechanics models, J Mech Phys Solids. 38 (1990) 379-404.

[44] Z. Hashin, The elastic moduli of heterogeneous materials, J. Appl. Mech. (1962) 143-150.

[45] Y. Huang, K. Hu, X. Wei, A. Chandra, A generalized self-consistent mechanics method for composite materials with multiphase inclusions, J. Mech. Phys. Solids. 42 (1994).

[46] T. Mori, K. Tanaka, Average stress in matrix and average elastic energy of materials with misfitting inclusions, Acta Metall. (1973) 1-4.

[47] I.O. Yaman, H.M. Aktan, N. Hearn, Active and non-active porosity in concrete part II: evaluation of existing models, Mater. Struct. 35 (2002) 110-116.

[48] G. Weng, Some elastic properties of reinforced solids, with special reference to isotropic ones containing spherical inclusions, Int. J. Eng. Sci. 22 (1984) 845-856.

[49] A. Norris, An examination of the Mori-Tanaka effective medium approximation for multiphase composites, J. Appl. Mech. 56 (1989) 83-88.

[50] G. Kuster, M. Toksöz, Velocity and attenuation of seismic waves in two-phase media: Part I. Theoretical formulations, Geophysics. 39 (1974) 587-606.

[51] J.G. Berryman, P.A. Berge, Critique of two explicit schemes for estimating elastic properties of multiphase composites, Mech. Mater. 22 (1996) 149-164. doi:10.1016/0167-6636(95)00035-6.

[52] R. McLaughlin, A study of the differential scheme for composite materials, Int. J. Eng. Sci. 15 (1977) 237-244.

[53] F.B. Hildebrand, Introduction to Numerical Analysis, Courier Corporation, 1987.

[54] K. Miled, K. Sab, R. Le Roy, Effective elastic properties of porous materials: Homogenization schemes vs experimental data, Mech. Res. Commun. 38 (2011) 131-135. doi:10.1016/j.mechrescom.2011.01.009.

[55] E. Garboczi, J. Berryman, Elastic moduli of a material containing composite inclusions: effective medium theory and finite element computations, Mech. Mater. 33 (2001).

[56] A.N. Norris, A differential scheme for the effective moduli of composites, Mech. Mater. 4 (1985) 116.

[57] M. Lutz, P. Monteiro, R. Zimmerman, Inhomogeneous interfacial transition zone model for the bulk modulus of mortar, Cem. Concr. Res. 27 (1997) 1113-1122.

[58] Z. Hashin, P.J.M. Monteiro, An inverse method to determine the elastic properties of the interphase between the aggregate and the cement paste, Cem. Concr. Res. 32 (2002) 1291-1300. doi:10.1016/S0008-8846(02)00792-5.

[59] J.A. Wang, J. Lubliner, P.J.M. Monteiro, Effect of ice formation on the elastic moduli of cement paste and mortar, Cem. Concr. Res. 18 (1988) 874-885.

[60] M. Königsberger, B. Pichler, C. Hellmich, Micromechanics of ITZ-aggregate interaction in concrete part I: Stress concentration, J. Am. Ceram. Soc. 97 (2014) 535-542. doi:10.1111/jace.12591.

[61] Z. Sun, E.J. Garboczi, S.P. Shah, Modeling the elastic properties of concrete composites: Experiment, differential effective medium theory, and numerical simulation, Cem. Concr. Compos. 29 (2007) 22-38. doi:10.1016/j.cemconcomp.2006.07.020. 
[62] C. Yang, Effect of the interfacial transition zone on the transport and the elastic properties of mortar, Mag. Concr. Res. 55 (2003) 305-312. doi:10.1680/macr.2003.55.4.305.

[63] C.Q. Li, X.Z. Zhou, J.J. Zheng, Thickness of interfacial transition zone and cement content profiles around aggregates, Mag. Concr. Res. 57 (2005) 397-406. doi:10.1680/macr.2005.57.7.397.

[64] M. Königsberger, B. Pichler, C. Hellmich, Micromechanics of ITZ-Aggregate Interaction in Concrete Part II: Strength Upscaling, J. Am. Ceram. Soc. 97 (2014) 543-551. doi:10.1111/jace.12606.

[65] J.J. Zheng, C.Q. Li, X.Z. Zhou, Characterization of microstructure of interfacial transition zone in concrete, ACl Mater. J. 102 (2005) 265-271.

[66] E.J. Garboczi, J.F. Douglas, Intrinsic conductivity of objects having arbitrary shape and conductivity, Phys. Rev. E. 53 (1996) 6169-6180. doi:10.1103/PhysRevE.53.6169.

[67] E.J. Garboczi, J.F. Douglas, R.B. Bohn, A hybrid finite element-analytical method for determining the intrinsic elastic moduli of particles having moderately extended shapes and a wide range of elastic properties, Mech. Mater. 38 (2006) 786-800. doi:10.1016/j.mechmat.2005.06.012.

[68] E.J. Garboczi, J.F. Douglas, Elastic moduli of composites containing a low concentration of complexshaped particles having a general property contrast with the matrix, Mech. Mater. 51 (2012) 5365. doi:10.1016/j.mechmat.2012.03.009.

[69] N. Neithalath, K. Ramamurthy, Microstructural investigations on aerated concrete, Cem. Concr. Res. 30 (2000) 457-464. doi:10.1016/S0008-8846(00)00199-X.

[70] H. Ma, Z. Li, Multi-aggregate approach for modeling interfacial transition zone in concrete, $\mathrm{ACI}$ Mater. J. 111 (2014).

http://www.concrete.org/Publications/InternationalConcreteAbstractsPortal.aspx?m=details\&i=51 686501 (accessed March 18, 2015).

[71] D.D. Bui, J. Hu, P. Stroeven, Particle size effect on the strength of rice husk ash blended gap-graded Portland cement concrete, Cem. Concr. Compos. 27 (2005) 357-366. doi:10.1016/j.cemconcomp.2004.05.002.

[72] P. Stroeven, M. Stroeven, Reconstructions by SPACE of the Interfacial Transition Zone, Cem. Concr. Compos. 23 (2001) 189-200. doi:10.1016/S0958-9465(00)00076-7.

[73] ASTM International, ASTM Standard C150/C150M. Standard specification of portland cement, ASTM International, West Conshohocken, PA, 2012.

[74] ASTM International, ASTM Standard C778. Standard specification for standard sand, ASTM International, West Conshohocken, PA, 2013.

[75] C.F. Ferraris, V.A. Hackley, A.I. Avilés, Measurement of particle size distribution in portland cement powder: Analysis of ASTM round robin studies, Cem. Concr. Aggreg. 26 (2004) 1-11.

[76] G. Gao, S. Moya, H. Lichtenfeld, A. Casoli, H. Fiedler, E. Donath, et al., The decomposition process of melamine formaldehyde cores: the key step in the fabrication of ultrathin polyelectrolyte multilayer capsules, Macromol. Mater. Eng. 286 (2001) 355-361.

[77] G. Ghosh, Dispersion-equation coefficients for the refractive index and birefringence of calcite and quartz crystals, Opt. Commun. 163 (1999) 95-102. doi:10.1016/S0030-4018(99)00091-7.

[78] ASTM International, ASTM Standard C305/C305M. Standard practice for mechanical mixing of hydraulic cement pastes and mortars of plastic consistency, ASTM International, West Conshohocken, PA, 2014.

[79] ASTM International, ASTM Standard C469/C469M. Standard test method for static modulus of elasticity and Poisson's ratio of concrete in compression, ASTM International, West Conshohocken, PA, 2014.

[80] M.M. Smadi, F.O. Slate, Microcracking of High and Normal Strength Concretes Under Short and Long-Term Loadings, ACI Mater. J. 86 (1989) 117-127. doi:10.14359/2264. 
[81] S.-H. Han, J.-K. Kim, Effect of temperature and age on the relationship between dynamic and static elastic modulus of concrete, Cem. Concr. Res. 34 (2004) 1219-1227. doi:10.1016/j.cemconres.2003.12.011.

[82] ASTM International, ASTM Standard C109/C109M. Compressive strength of hydraulic cement mortar (Using 2-in. or [50-mm] cube specimens), ASTM International, West Conshohocken, PA, 2014.

[83] M. Hossain, C. Ketata, M. Islam, Experimental study of physical and mechanical properties of natural and synthetic waxes using uniaxial compressive strength test, in: Proc. Third Int. Conf. Model. Simul. Appl. Optim., 2009: pp. 1-5.

[84] X. Chen, S. Zhang, G.J. Wagner, W. Ding, R.S. Ruoff, Mechanical resonance of quartz microfibers and boundary condition effects, J. Appl. Phys. 95 (2004) 4823-4828. doi:10.1063/1.1697635.

[85] ACl 318, Building Code Requirements for Reinforced Concrete, Am Conc Inst, Detroit, MI, 2002.

[86] T. Hirsch, Modulus of elasticity of concrete affected by elastic moduli of cement paste matrix and aggregate, ACl J. Proc. 59 (1962) 427-452.

[87] R.W. Rice, Porosity of Ceramics: Properties and Applications, CRC Press, 1998.

[88] R.W. Rice, Evaluating Porosity Parameters for Porosity-Property Relations, J. Am. Ceram. Soc. 76 (1993) 1801-1808. doi:10.1111/j.1151-2916.1993.tb06650.x.

[89] R.W. Rice, Evaluation and extension of physical property-porosity models based on minimum solid area, J. Mater. Sci. 31 (1996) 102-118. doi:10.1007/BF00355133.

[90] S. Torquato, Random Heterogeneous Materials: Microstructure and Macroscopic Properties, Springer Science \& Business Media, 2002.

[91] D.P. Bentz, Three-Dimensional Computer Simulation of Portland Cement Hydration and Microstructure Development, J. Am. Ceram. Soc. 80 (1997) 3-21. doi:10.1111/j.11512916.1997.tb02785.x.

[92] A. Kumar, T. Oey, S. Kim, D. Thomas, S. Badran, J. Li, et al., Simple methods to estimate the influence of limestone fillers on reaction and property evolution in cementitious materials, Cem. Concr. Compos. 42 (2013) 20-29. doi:10.1016/j.cemconcomp.2013.05.002.

[93] F. de Larrard, T. Sedran, Optimization of ultra-high-performance concrete by the use of a packing model, Cem. Concr. Res. 24 (1994) 997-1009. doi:10.1016/0008-8846(94)90022-1.

[94] K.L. Scrivener, K.M. Nemati, The percolation of pore space in the cement paste/aggregate interfacial zone of concrete, Cem. Concr. Res. 26 (1996) 35-40.

[95] A. Elsharief, M.D. Cohen, J. Olek, Influence of aggregate size, water cement ratio and age on the microstructure of the interfacial transition zone, Cem. Concr. Res. 33 (2003) 1837-1849. doi:10.1016/S0008-8846(03)00205-9.

[96] R.P. Khatri, V. Sirivivatnanon, W. Gross, Effect of different supplementary cementitious materials on mechanical properties of high performance concrete, Cem. Concr. Res. 25 (1995) 209-220. doi:10.1016/0008-8846(94)00128-L. 